Pacific

Journal of

Mathematics

GRADIENT ESTIMATES AND ENTROPY FORMULAE OF POROUS MEDIUM AND FAST DIFFUSION EQUATIONS FOR THE WITTEN LAPLACIAN

GUANGYUE HUANG AND HAIZHONG Li

Volume $268 \quad$ No. 1

March 2014 


\title{
GRADIENT ESTIMATES AND ENTROPY FORMULAE OF POROUS MEDIUM AND FAST DIFFUSION EQUATIONS FOR THE WITTEN LAPLACIAN
}

\author{
GuANGYUE HUANG AND HAIZHONG Li
}

We study gradient estimates for the positive solutions of the porous medium equations and the fast diffusion equations

$$
u_{t}=\Delta_{\phi}\left(u^{p}\right)
$$

associated with the Witten Laplacian on Riemannian manifolds. Under the assumption that the $\boldsymbol{m}$-dimensional Bakry-Emery Ricci curvature is bounded from below, we obtain some gradient estimates which generalize some previous results of Lu et. al. and Huang et. al. As applications, several parabolic Harnack inequalities are obtained. Moreover, inspired by X.-D. Li's work, we also extend the entropy formulae introduced by $\mathrm{Lu}$ et. al. to the porous medium equations and the fast diffusion equations associated with the Witten Laplacian. We prove some monotonicity theorems for such entropy on compact Riemannian manifolds with nonnegative $m$ dimensional Bakry-Emery Ricci curvature.

\section{Introduction}

Let $\left(M^{n}, g\right)$ be an $n$-dimensional complete Riemannian manifold. P. Li and Yau [1986] considered positive solutions of the heat equation

$$
u_{t}=\Delta u
$$

and proved the following gradient estimates.

Theorem A [Li and Yau 1986]. Let $\left(M^{n}, g\right)$ be a complete Riemannian manifold with $\operatorname{Ric}\left(B_{p}(2 R)\right) \geq-K$, where $\operatorname{Ric}\left(B_{p}(2 R)\right)$ denotes the Ricci curvature on the geodesic ball $B_{p}(2 R)$ with radius $2 R$ and $K$ is a nonnegative constant. Let $u$ be a

Huang was supported by the NSFC (grant numbers 11001076, 11171091, 11371018). Li was supported by the NSFC (grant number 11271214).

MSC2010: primary 35B45; secondary 35K55.

Keywords: porous medium equation, fast diffusion equation, entropy formulae, Witten Laplacian. 
positive solution of $(1-1)$ on $B_{p}(2 R) \times[0, T]$. Then, on $B_{p}(R)$, we have

$$
\frac{|\nabla u|^{2}}{u^{2}}-\alpha \frac{u_{t}}{u} \leq \frac{C(n) \alpha^{2}}{R^{2}}\left(\frac{\alpha^{2}}{\alpha-1}+\sqrt{K} R\right)+\frac{n \alpha^{2} K}{2(\alpha-1)}+\frac{n \alpha^{2}}{2 t},
$$

where $\alpha>1$ is a constant and $C(n)$ is a constant depending only on $n$. Moreover, taking $R \rightarrow \infty,(1-2)$ yields the following estimate on $\left(M^{n}, g\right)$ :

$$
\frac{|\nabla u|^{2}}{u^{2}}-\alpha \frac{u_{t}}{u} \leq \frac{n \alpha^{2} K}{2(\alpha-1)}+\frac{n \alpha^{2}}{2 t} .
$$

J. F. Li and X. J. Xu [2011] obtained new Li-Yau-type gradient estimates for positive solutions of the heat equation (1-1) on complete Riemannian manifolds. For related research and some improvements on Li-Yau-type gradient estimates of (1-1), see [Yau 1994; 1995; Bakry and Qian 1999; Hamilton 1993; Li 2005; Davies 1989]. The equation

$$
u_{t}=\Delta\left(u^{p}\right)
$$

with $p>1$ is called the porous medium equation, which is a nonlinear extension of the classical heat equation. For various values of $p>1$, it has appeared in different applications to model diffusive phenomena (see [Vázquez 2007; Aronson and Bénilan 1979; Lu et al. 2009] and the references therein). Equation (1-4) with $p \in(0,1)$ is called the fast diffusion equation, which appears in plasma physics and in geometric flows. However, there are remarkable differences between the porous medium equations and the fast diffusion equation; see [Vázquez 2006; Daskalopoulos and Kenig 2007]. For the study of gradient estimates of (1-4), see [Huang et al. 2013; Aronson and Bénilan 1979; Vázquez 2007; Xu 2012].

$\mathrm{Lu}, \mathrm{Ni}$, Vázquez, and Villani studied gradient estimates of (1-4) and proved the following results.

Theorem B [Lu et al. 2009, Theorem 3.3]. Let $\left(M^{n}, g\right)$ be a complete Riemannian manifold with $\operatorname{Ric}\left(B_{p}(2 R)\right) \geq-K$, where $\operatorname{Ric}\left(B_{p}(2 R)\right)$ denotes the Ricci curvature on the geodesic ball $B_{p}(2 R)$ with radius $2 R$ and $K$ is a nonnegative constant. Let $u$ be a positive solution to (1-4) with $p>1$. Let $v=(p /(p-1)) u^{p-1}$ and $M=(p-1) \max _{B_{p}(2 R) \times[0, T]} v$. Then, for any $\alpha>1$, on $B_{p}(R)$, we have

$$
\begin{aligned}
& \frac{|\nabla v|^{2}}{v}-\alpha \frac{v_{t}}{v} \\
& \quad \leq \frac{C(n) M a \alpha^{2}}{R^{2}}\left(\frac{\alpha^{2}}{\alpha-1} \frac{a p^{2}}{p-1}+(1+\sqrt{K} R)\right)+\frac{\alpha^{2}}{\alpha-1} a M K+\frac{a \alpha^{2}}{t},
\end{aligned}
$$


where $a=n(p-1) /(n(p-1)+2)$. Moreover, taking $R \rightarrow \infty,(1-5)$ yields the following estimate on $\left(M^{n}, g\right)$ :

$$
\frac{|\nabla v|^{2}}{v}-\alpha \frac{v_{t}}{v} \leq \frac{\alpha^{2}}{\alpha-1} a M K+\frac{a \alpha^{2}}{t} .
$$

Now we rewrite the inequality (1-6) as

$$
|\nabla v|^{2}-\alpha v_{t} \leq \frac{\alpha^{2}}{\alpha-1} a M K v+\frac{a \alpha^{2} v}{t} .
$$

Since $(p-1) v=p u^{p-1}$, we have $(p-1) v \rightarrow 1$ as $p \rightarrow 1$. As $p \rightarrow 1$, we have $M \rightarrow 1$,

$$
|\nabla v|^{2} \rightarrow \frac{|\nabla u|^{2}}{u^{2}}, \quad v_{t} \rightarrow \frac{u_{t}}{u}, \quad a v \rightarrow \frac{n}{2}
$$

Consequently, (1-7) becomes Li and Yau's inequality (1-3). Therefore, for a complete noncompact Riemannian manifold $\left(M^{n}, g\right)$, estimate (1-6) in the result of $\mathrm{Lu}$, $\mathrm{Ni}$, Vázquez and Villani reduces to estimate (1-3) when $p \rightarrow 1$.

Let $\phi \in C^{2}\left(M^{n}\right)$. The Witten Laplacian associated with $\phi$ is defined by

$$
\Delta_{\phi}=\Delta-\nabla \phi \cdot \nabla
$$

which is symmetric with respect to the $L^{2}\left(M^{n}\right)$ inner product under the weighted measure

$$
d \mu=e^{-\phi} d v
$$

that is,

$$
\int_{M^{n}} u \Delta_{\phi} v d \mu=-\int_{M^{n}} \nabla u \nabla v d \mu=\int_{M^{n}} v \Delta_{\phi} u d \mu \quad \text { for all } u, v \in C_{0}^{\infty}\left(M^{n}\right) .
$$

Following [Bakry and Émery 1985; Bakry 1994; Li 2005; Wei and Wylie 2009], we introduce the $m$-dimensional Bakry-Emery Ricci curvature associated with the Witten Laplacian by

$$
\operatorname{Ric}_{\phi}^{m}=\operatorname{Ric}+\nabla^{2} \phi-\frac{1}{m-n} d \phi \otimes d \phi,
$$

where $m \geq n$ is a constant and $m=n$ if and only if $\phi$ is a constant. Define

$$
\operatorname{Ric}_{\phi}=\operatorname{Ric}+\nabla^{2} \phi .
$$

Then $\operatorname{Ric}_{\phi}$ can be seen as the $\infty$-dimensional Bakry-Emery Ricci curvature. In this paper, we study the following equation associated with the Witten Laplacian:

$$
u_{t}=\Delta_{\phi}\left(u^{p}\right)
$$

with $p>0$ and $p \neq 1$. For $p>1$ and $p \in(0,1)$, we derive an analogue of the estimates of Lu, Ni, Vázquez, and Villani and a Davies-type estimate. Moreover, 
for $p>1$, we obtain a Hamilton-type estimate and an analogue of the estimates of $\mathrm{Li}$ and $\mathrm{Xu}$. In particular, our results generalize the ones in [Huang et al. 2013].

First we consider gradient estimates of (1-8) under the assumption that the $m$ dimensional Bakry-Emery Ricci curvature is bounded from below, and obtain the following results. We set once and for all

$$
\tilde{a}=\frac{m(p-1)}{m(p-1)+2} .
$$

Theorem 1.1. Let $\left(M^{n}, g\right)$ be a complete Riemannian manifold with

$$
\operatorname{Ric}_{\phi}^{m}\left(B_{p}(2 R)\right) \geq-K,
$$

where $\operatorname{Ric}_{\phi}^{m}\left(B_{p}(2 R)\right)$ denotes the m-dimensional Bakry-Emery Ricci curvature on the geodesic ball $B_{p}(2 R)$ with radius $2 R$, and $K$ is a nonnegative constant. Let $u$ be a positive solution to the porous medium equation (1-8) with $p>1$. Let $v=(p /(p-1)) u^{p-1}$ and $M=(p-1) \max _{B_{p}(2 R) \times[0, T]} v$. Then, for any $\alpha>1$, on $B_{p}(R)$, we have

$$
\begin{aligned}
\frac{|\nabla v|^{2}}{v}-\alpha \frac{v_{t}}{v} \leq \tilde{a} \alpha^{2} M \frac{C(m)}{R^{2}}\left(\frac{\alpha^{2}}{\alpha-1} \frac{\tilde{a} p^{2}}{p-1}+1+\sqrt{K} R \operatorname{coth}(\sqrt{K} R)\right) & \\
& +\frac{\alpha^{2}}{(\alpha-1)} \tilde{a} M K+\frac{\tilde{a} \alpha^{2}}{t} .
\end{aligned}
$$

Taking $R \rightarrow \infty$, we thus obtain the following estimate on $\left(M^{n}, g\right)$ :

$$
\frac{|\nabla v|^{2}}{v}-\alpha \frac{v_{t}}{v} \leq \frac{\alpha^{2}}{\alpha-1} \tilde{a} M K+\frac{\tilde{a} \alpha^{2}}{t} .
$$

Corollary 1.2. Let $\left(M^{n}, g\right)$ be a complete Riemannian manifold with $\mathrm{Ric}_{\phi}^{m} \geq-K$, where $K$ is a nonnegative constant. Let $u$ be a positive solution to (1-8) with $p>1$. Set

$$
v=\frac{p}{p-1} u^{p-1}, \quad M=(p-1) \sup _{M^{n} \times[0, T]} v, \quad \widetilde{M}=\inf _{M^{n} \times[0, T]} v .
$$

Then, for any $x_{1}, x_{2} \in M^{n}, 0<t_{1}<t_{2}<T, \alpha>1$, we have

$$
v\left(x_{1}, t_{1}\right) \leq v\left(x_{2}, t_{2}\right)\left(\frac{t_{2}}{t_{1}}\right)^{\tilde{a} \alpha} \exp \left(\frac{\alpha \operatorname{dist}^{2}\left(x_{2}, x_{1}\right)}{4 \widetilde{M}\left(t_{2}-t_{1}\right)}+\frac{\alpha}{\alpha-1} \tilde{a} M K\left(t_{2}-t_{1}\right)\right),
$$

where $\operatorname{dist}\left(x_{2}, x_{1}\right)$ is the distance between $x_{1}$ and $x_{2}$.

Theorem 1.3. Let $\left(M^{n}, g\right)$ and $K$ be as in Theorem 1.1. Let $u$ be a positive solution to the fast diffusion equation (1-8) with $p \in(1-2 / m, 1)$. Set

$$
v=\frac{p}{p-1} u^{p-1}, \quad M=(1-p) \max _{B_{p}(2 R) \times[0, T]}(-v) .
$$


Then, for any $0<\alpha<1$, we have on $B_{p}(R)$

$(1-11)-\frac{|\nabla v|^{2}}{v}+\alpha \frac{v_{t}}{v}$

$$
\begin{array}{r}
\leq \frac{(-\tilde{a}) \alpha^{2} M}{A\left(\varepsilon_{1}, \varepsilon_{2}\right)} \frac{C(m)}{R^{2}}\left(\frac{(-\tilde{a}) \alpha^{2} p^{2}}{2 \varepsilon_{2}(1-\tilde{a})(1-\alpha)(1-p)}+1+\sqrt{K} R \operatorname{coth}(\sqrt{K} R)\right) \\
\quad+\frac{(-\tilde{a}) \alpha^{2} M K}{\sqrt{\varepsilon_{1}(1-\alpha)(1-\alpha-\tilde{a}) A\left(\varepsilon_{1}, \varepsilon_{2}\right)}}+\frac{(-\tilde{a}) \alpha^{2}}{A\left(\varepsilon_{1}, \varepsilon_{2}\right) t},
\end{array}
$$

where $\varepsilon_{1}, \varepsilon_{2} \in(0,1)$ are positive constants satisfying

$$
A\left(\varepsilon_{1}, \varepsilon_{2}\right):=[1-\tilde{a}(1-\alpha)]-\frac{\left(1+\varepsilon_{2}\right)^{2}(1-\tilde{a})^{2}(1-\alpha)}{\left(1-\varepsilon_{1}\right)(1-\alpha-\tilde{a})}>0 .
$$

Taking $R \rightarrow \infty$ and $\alpha \rightarrow 1$, we thus obtain the following estimate on $\left(M^{n}, g\right)$ with $\operatorname{Ric}_{\phi}^{m} \geq 0$ :

$$
-\frac{|\nabla v|^{2}}{v}+\frac{v_{t}}{v} \leq-\frac{\tilde{a}}{t}
$$

Corollary 1.4. Let $\left(M^{n}, g\right)$ be a complete Riemannian manifold with $\mathrm{Ric}_{\phi}^{m} \geq 0$. Let $u$ be a positive solution to (1-8) with $p \in(1-2 / m, 1)$. Set

$$
v=\frac{p}{p-1} u^{p-1}, \quad M=(1-p) \sup _{M^{n} \times[0, T]}(-v), \quad \widetilde{M}=\inf _{M^{n} \times[0, T]}(-v) .
$$

Then, for any $x_{1}, x_{2} \in M^{n}$ and $0<t_{1}<t_{2}<T$, we have

$$
-v\left(x_{2}, t_{2}\right) \leq-v\left(x_{1}, t_{1}\right)\left(\frac{t_{2}}{t_{1}}\right)^{-\tilde{a}} \exp \frac{\operatorname{dist}^{2}\left(x_{2}, x_{1}\right)}{4 \widetilde{M}\left(t_{2}-t_{1}\right)},
$$

where $\operatorname{dist}\left(x_{2}, x_{1}\right)$ is the distance between $x_{1}$ and $x_{2}$.

Remark 1.5. Clearly, our estimate (1-10) reduces to (1-6) (see [Lu et al. 2009]) by letting $m=n$. Moreover, for $p \in(0,1)$, [Lu et al. 2009, Theorem 4.1] can be obtained from our Theorem 1.3 by taking $m=n$.

Theorem 1.6. Let $\left(M^{n}, g\right)$ and $K$ be as in Theorem 1.1. Let $u$ be a positive solution to the fast diffusion equation (1-8) with $p \in(1-2 / m, 1)$. Set

$$
v=\frac{p}{p-1} u^{p-1}, \quad M=(1-p) \max _{B_{p}(2 R) \times[0, T]}(-v) .
$$

Then, for any $0<\alpha<1$, we have on $B_{p}(R)$ 


$$
\begin{aligned}
-\frac{|\nabla v|^{2}}{v}+\alpha \frac{v_{t}}{v} \leq\{ & C(\tilde{a}, \alpha) \frac{p}{\sqrt{1-p}} \sqrt{M} \frac{C}{R} \\
+ & {\left[\left(\frac{\alpha^{2}}{2(1-\alpha)}+2(1-\tilde{a})\right) M K+\frac{1-\alpha-\tilde{a}}{t}\right.} \\
& \left.\left.+(1-p)(1-\alpha-\tilde{a}) M \frac{C(m)}{R^{2}}(1+\sqrt{K} R \operatorname{coth}(\sqrt{K} R))\right]^{\frac{1}{2}}\right\}^{2} .
\end{aligned}
$$

Taking $R \rightarrow \infty$, we thus obtain the following estimate on $\left(M^{n}, g\right)$ :

$$
-\frac{|\nabla v|^{2}}{v}+\alpha \frac{v_{t}}{v} \leq\left(\frac{\alpha^{2}}{2(1-\alpha)}+2(1-\tilde{a})\right) M K+\frac{1-\alpha-\tilde{a}}{t} .
$$

Corollary 1.7. Let $\left(M^{n}, g\right)$ be a complete Riemannian manifold with $\operatorname{Ric}_{\phi}^{m} \geq-K$, where $K$ is a nonnegative constant. Let $u$ be a positive solution to (1-8) with $p \in(1-2 / m, 1)$. Let

$$
v=\frac{p}{p-1} u^{p-1}, \quad M=(1-p) \sup _{M^{n} \times[0, T]}(-v), \quad \widetilde{M}=\inf _{M^{n} \times[0, T]}(-v) .
$$

Then, for any $x_{1}, x_{2} \in M^{n}, 0<t_{1}<t_{2}<T, 0<\alpha<1$, we have

$$
\begin{aligned}
-v\left(x_{2}, t_{2}\right) \leq-v\left(x_{1}, t_{1}\right) & \left(\frac{t_{2}}{t_{1}}\right)^{(1-\alpha-\tilde{a}) / \alpha} \\
& \times \exp \left(\frac{\alpha \operatorname{dist}^{2}\left(x_{2}, x_{1}\right)}{4 \widetilde{M}\left(t_{2}-t_{1}\right)}+\left(\frac{\alpha}{2(1-\alpha)}+\frac{2(1-\tilde{a})}{\alpha}\right) M K\left(t_{2}-t_{1}\right)\right)
\end{aligned}
$$

where $\operatorname{dist}\left(x_{2}, x_{1}\right)$ is the distance between $x_{1}$ and $x_{2}$.

Remark 1.8. For complete Riemannian manifolds with $p \in(0,1)$, Corollary 4.2 of [Lu et al. 2009] shows that, if Ric $\geq 0$, then

$$
-\frac{|\nabla v|^{2}}{v}+\frac{v_{t}}{v} \leq-\frac{a}{t}
$$

while if Ric $\geq-K$ and $0<\alpha<1$, then, for any $\varepsilon>0$ satisfying

$$
C(a, \alpha, \varepsilon):=1+(-a)(1-\alpha)-\frac{(1-\alpha)(1-a)^{2}}{(1-\alpha)-a-(1-\alpha) \varepsilon^{2}}>0,
$$

we have

$$
-\frac{|\nabla v|^{2}}{v}+\alpha \frac{v_{t}}{v} \leq \frac{(-a) \alpha^{2}}{C(a, \alpha, \varepsilon)}\left(\frac{1}{t}+\frac{\sqrt{C(a, \alpha, \varepsilon)}}{(1-\alpha) \varepsilon} M K\right) .
$$

Obviously, our estimate (1-14) reduces to (1-15) when $m=n$ and $\alpha \rightarrow 1$. Moreover, (1-14) is independent of $\varepsilon$. 
Denote by $R$ the scalar curvature of the metric $g$. Perelman [2002] introduced the $\mathcal{W}$-entropy functional as

$$
\mathscr{W}(g, f, \tau)=\int_{M^{n}}\left(\tau\left(R+|\nabla f|^{2}\right)+f-n\right) \frac{e^{-f}}{(4 \pi \tau)^{n / 2}} d v,
$$

where $\tau$ is a positive scale parameter and $f \in C^{\infty}\left(M^{n}\right)$ satisfies

$$
\int_{M^{n}} \frac{e^{-f}}{(4 \pi \tau)^{n / 2}} d v=1
$$

By [Perelman 2002], we know that the $\mathcal{W}$-entropy is monotone increasing under the Ricci flow, and its critical points are given by gradient shrinking solitons. Ni [2004a; 2004b] considered the $\mathcal{W}$-entropy for the linear heat equation

$$
u_{\tau}=\Delta u
$$

on complete Riemannian manifolds. More precisely, Ni [2004a] introduced the W-entropy associated with (1-18) by

$$
\mathcal{W}(g, f, \tau)=\int_{M^{n}}\left[\tau|\nabla f|^{2}+f-n\right] \frac{e^{-f}}{(4 \pi \tau)^{n / 2}} d v,
$$
where $u=\frac{e^{-f}}{(4 \pi \tau)^{n / 2}}$ is a positive solution to (1-18) and $\int_{M^{n}} u d v=1$, and proved
that

$$
\frac{d}{d \tau} \mathcal{W}(g, f, \tau)=-2 \int_{M^{n}} \tau\left(\left|\nabla^{2} f-\frac{g}{2 \tau}\right|^{2}+\operatorname{Ric}(\nabla f, \nabla f)\right) u d v .
$$

Thus, if the Ricci curvature is nonnegative, the $\mathcal{W}$-entropy defined by (1-19) is nonincreasing on complete Riemannian manifolds. For research on the monotonicity of W-entropy for other geometric heat flows on Riemannian manifolds, see [Kotschwar and Ni 2009; Ecker 2007; Ni 2004a; 2004b; Lu et al. 2009]. X.-D. Li [2011; 2012; 2013] studied the $\mathscr{W}_{m}$-entropy associated with the Witten Laplacian to the linear heat equation

$$
u_{\tau}=\Delta_{\phi} u
$$

on complete Riemannian manifolds satisfying the $\mu$-bounded geometry condition. More precisely, [Li 2012] introduced the $\mathcal{W}_{m}$-entropy associated with (1-21) by

$$
\mathscr{W}_{m}(g, f, \tau)=\int_{M^{n}}\left[\tau|\nabla f|^{2}+f-m\right] \frac{e^{-f}}{(4 \pi \tau)^{m / 2}} d \mu,
$$

where $u=\frac{e^{-f}}{(4 \pi \tau)^{m / 2}}$ is a positive solution to (1-21), and proved that if there exist 
two constants $m>n$ and $K \geq 0$ such that $\operatorname{Ric}_{\phi}^{m} \geq-K$, then

$$
\begin{aligned}
\frac{d}{d \tau} W_{m}(g, f, \tau)=-2 \int_{M^{n}} \tau\left(\mid \nabla^{2} f-\right. & \left.\left.\frac{g}{2 \tau}\right|^{2}+\operatorname{Ric}_{\phi}^{m}(\nabla f, \nabla f)\right) u d \mu \\
& -\frac{2}{m-n} \int_{M^{n}} \tau\left(\nabla \phi \nabla f+\frac{m-n}{2 \tau}\right)^{2} u d \mu .
\end{aligned}
$$

Thus, if $\operatorname{Ric}_{\phi}^{m} \geq 0$, then $\mathscr{W}_{m}(g, f, \tau)$ is nonincreasing along the heat equation (1-21). For the study of the Witten Laplacian associated with the $m$-dimensional BakryEmery Ricci curvature on complete Riemannian manifolds, see [Wei and Wylie 2009; Wang 2004; 1997; Qian 1998; 1997; Ni 2002; Li 2005; Fang et al. 2009; Bakry and Qian 2005; Bakry 1994; Bakry and Émery 1985]. Let $u$ be a positive solution to (1-4), and let $v=(p /(p-1)) u^{p-1}$. Lu et. al. [2009] introduced

$$
\mathcal{N}_{p}(g, u, t)=-t^{a} \int_{M^{n}} u v d v
$$

and

$$
\mathscr{W}_{p}(g, u, t)=\frac{d}{d t}\left[t \mathcal{N}_{p}(g, u, t)\right]=t^{a+1} \int_{M^{n}}\left(p \frac{|\nabla v|^{2}}{v}-\frac{a+1}{t}\right) u v d v,
$$

where $a=\frac{n(p-1)}{n(p-1)+2}$. They proved that if $M^{n}$ is compact,

$$
\begin{aligned}
& \frac{d}{d t} W_{p}(g, u, t) \\
& =-2(p-1) t^{a+1} \int_{M^{n}}\left(\left|\nabla^{2} v+\frac{g}{[n(p-1)+2] t}\right|^{2}+\operatorname{Ric}(\nabla v, \nabla v)\right) u v d v \\
& \quad-2 t^{a+1} \int_{M^{n}}\left((p-1) \Delta v+\frac{a}{t}\right)^{2} u v d v .
\end{aligned}
$$

In particular, if the Ricci curvature is nonnegative, the entropy defined in (1-24) is nonincreasing on compact Riemannian manifolds when $p>1$. For $p<1$, using the Cauchy-Schwarz inequality, they proved from (1-25) that

$$
\text { (1-26) } \begin{aligned}
& \frac{d}{d t} \mathscr{W}_{p}(g, u, t) \\
\leq & -2 t^{a+1} \int_{M^{n}}\left[\frac{n(p-1)+1}{n(p-1)}\left((p-1) \Delta v+\frac{a}{t}\right)^{2}+(p-1) \operatorname{Ric}(\nabla v, \nabla v)\right] u v d v .
\end{aligned}
$$

Clearly, if the Ricci curvature is nonnegative and $p \in(1-1 / n, 1)$, then (1-26) shows that $(d / d t)^{\mathscr{W}} \mathcal{W}_{p}(g, u, t) \leq 0$ and the entropy defined in (1-24) is nonincreasing on compact Riemannian manifolds. 
Inspired by [Li 2012], in this paper we also study the $\mathcal{W}_{p, m}$-entropy for (1-8) associated with the Witten Laplacian on compact Riemannian manifolds with $p>0$ and $p \neq 1$. First we define

$$
\mathcal{N}_{p, m}(g, u, t)=-t^{\tilde{a}} \int_{M^{n}} u v d \mu,
$$

where the $\mathcal{W}_{p, m}$-entropy is defined by

$$
\mathcal{W}_{p, m}(g, u, t)=\frac{d}{d t}\left[t \mathcal{N}_{p, m}(g, u, t)\right],
$$

When the $m$-dimensional Bakry-Emery Ricci curvature is bounded from below, we prove the following.

Theorem 1.9. Let $\left(M^{n}, g\right)$ be a compact Riemannian manifold. If $u$ is a positive solution to the porous medium equation (1-8) with $p>1$, then

$$
\frac{d}{d t} \mathcal{N}_{p, m}(g, u, t)=-t^{\tilde{a}} \int_{M^{n}}\left((p-1) \Delta_{\phi} v+\frac{\tilde{a}}{t}\right) u v d \mu,
$$

where $v=(p /(p-1)) u^{p-1}$. In particular, if $\operatorname{Ric}_{\phi}^{m} \geq 0$, then $\mathcal{N}_{p, m}(g, u, t)$ is nonincreasing in $t$. Moreover,

$$
\mathscr{W}_{p, m}(g, u, t)=t^{\tilde{a}+1} \int_{M^{n}}\left(p \frac{|\nabla v|^{2}}{v}-\frac{\tilde{a}+1}{t}\right) u v d \mu
$$

and

$$
\begin{aligned}
& \frac{d}{d t} \mathscr{W}_{p, m}(g, u, t) \\
& =-2(p-1) t^{\tilde{a}+1} \int_{M^{n}}\left(\left|\nabla^{2} v+\frac{g}{[m(p-1)+2] t}\right|^{2}+\operatorname{Ric}_{\phi}^{m}(\nabla v, \nabla v)\right. \\
& \left.\quad+\frac{1}{m-n}\left|\nabla \phi \nabla v-\frac{m-n}{[m(p-1)+2] t}\right|^{2}\right) u v d \mu \\
& \quad-2 t^{\tilde{a}+1} \int_{M^{n}}\left|(p-1) \Delta_{\phi} v+\frac{\tilde{a}}{t}\right|^{2} u v d \mu .
\end{aligned}
$$

In particular, if $\operatorname{Ric}_{\phi}^{m} \geq 0$, then $\mathcal{W}_{p, m}(g, u, t)$ is nonincreasing in $t$.

Theorem 1.10. Let $\left(M^{n}, g\right)$ be a compact Riemannian manifold. If $u$ is a positive solution to the fast diffusion equation (1-8) with $p \in(0,1)$, then

$$
\frac{d}{d t} \mathcal{N}_{p, m}(g, u, t)=-t^{\tilde{a}} \int_{M^{n}}\left((p-1) \Delta_{\phi} v+\frac{\tilde{a}}{t}\right) u v d \mu,
$$

where $v=\frac{p}{p-1} u^{p-1}$. In particular, if $\operatorname{Ric}_{\phi}^{m} \geq 0$ and $p \in(1-2 / m, 1)$, then 
$\mathcal{N}_{p, m}(g, u, t)$ is nonincreasing in $t$. Moreover, we have

$$
\mathscr{W}_{p, m}(g, u, t)=t^{\tilde{a}+1} \int_{M^{n}}\left(p \frac{|\nabla v|^{2}}{v}-\frac{\tilde{a}+1}{t}\right) u v d \mu,
$$

and, for any positive constant $\varepsilon \geq m-n$ and $1-\frac{1}{n+\varepsilon} \leq p \leq 1-\frac{m-n}{m \varepsilon}$,

(1-34) $\frac{d}{d t} W_{p, m}(g, u, t)$

$$
\begin{aligned}
& \leq 2 t^{\tilde{a}+1} \int_{M^{n}}((1-p) \operatorname{Ric}_{\phi}^{m}(\nabla v, \nabla v)+\left(\frac{1-n(1-p)}{n(1-p)}-\frac{\varepsilon}{n}\right)\left|(p-1) \Delta_{\phi} v+\frac{\tilde{a}}{t}\right|^{2} \\
&\left.+\left(\frac{m(1-p)}{n(m-n)}-\frac{1}{n \varepsilon}\right)\left|\nabla \phi \nabla v-\frac{m-n}{[m(p-1)+2] t}\right|^{2}\right) u v d \mu .
\end{aligned}
$$

In particular, if $\operatorname{Ric}_{\phi}^{m} \geq 0$, then $W_{p, m}(g, u, t)$ is nonincreasing in $t$.

Remark 1.11. If $m=n$, we see that $\phi$ is a constant. Then (1-31) becomes [Lu et al. 2009, (5.6)]. By letting $m=n$ and $\varepsilon \rightarrow 0$, (1-34) becomes (1-26), which is [Lu et al. 2009, Corollary 5.10].

Remark 1.12. After we submitted our paper, the referee pointed out to us [ $\mathrm{Li}$ and Li 2013; Wang and Chen 2013; Wang et al. 2013], in which some related problems are studied. Specifically, S. Li and X.-D. Li [2013] derived the $W$-entropy formula for the Witten Laplacian on manifolds with time dependent metrics and potentials. Wang and Chen [2013] obtained Aronson-Bénilan-type estimates for the porous medium equations associated with the Witten Laplacian. Wang, Yang, and Chen [Wang et al. 2013] studied the weighted $p$-Laplacian heat equation and proved an optimal gradient estimate and the $W$-entropy monotonicity formula, which generalized the results of [Kotschwar and Ni 2009]. We note that the first version of this paper was posted on arXiv (1203.5482) on March 25 of 2012.

\section{Proofs of Theorems 1.1 and 1.3}

Let $v=(p /(p-1)) u^{p-1}$. By virtue of (1-8), we have $v_{t}=(p-1) v \Delta_{\phi} v+|\nabla v|^{2}$, which is equivalent to

$$
\frac{v_{t}}{v}=(p-1) \Delta_{\phi} v+\frac{|\nabla v|^{2}}{v} .
$$

As in [Lu et al. 2009], we introduce the differential operator

$$
\mathscr{L}=\partial_{t}-(p-1) v \Delta_{\phi} .
$$


Lemma 2.1. Let $F=\frac{|\nabla v|^{2}}{v}-\alpha \frac{v_{t}}{v}-\varphi$, where $\alpha=\alpha(t)$ and $\varphi=\varphi(t)$ are functions of $t$. Set

$L_{0}(F)=$

$-\frac{1}{\tilde{a}}\left[(p-1) \Delta_{\phi} v\right]^{2}-2(p-1) \operatorname{Ric}_{\phi}^{m}(\nabla v, \nabla v)+2 p \nabla v \nabla F+(1-\alpha)\left(\frac{v_{t}}{v}\right)^{2}-\alpha^{\prime} \frac{v_{t}}{v}-\varphi^{\prime}$.

(1) If $p>1$, then $\mathscr{L}(F) \leq L_{0}(F)$.

(2) If $p \in(0,1)$, then $\mathscr{L}(F) \geq L_{0}(F)$.

Proof. We only give the proof for the case where $p>1$; the other case is similar. By a direct calculation, we have

(2-3) $\mathscr{L}\left(\frac{f}{g}\right)=\frac{1}{g} \mathscr{L}(f)-\frac{f}{g^{2}} \mathscr{L}(g)+2(p-1) v \nabla \frac{f}{g} \nabla \log g$ for all $f, g \in C^{\infty}(M)$.

Using (2-1), we obtain

$$
\mathscr{L}\left(v_{t}\right)=(p-1) v_{t} \Delta_{\phi} v+2 \nabla v \nabla v_{t} .
$$

It is well known that, for the $m$-dimensional Bakry-Emery Ricci curvature, we have the following Bochner formula (for the elementary proof, see [Ledoux 2000; Li 2005]):

$$
\begin{aligned}
\frac{1}{2} \Delta_{\phi}\left(|\nabla w|^{2}\right) & =\left|\nabla^{2} w\right|^{2}+\nabla w \nabla \Delta_{\phi} w+\operatorname{Ric}_{\phi}(\nabla w, \nabla w) \\
& \geq \frac{1}{n}|\Delta w|^{2}+\nabla w \nabla \Delta_{\phi} w+\operatorname{Ric}_{\phi}(\nabla w, \nabla w) \\
& \geq \frac{1}{m}\left|\Delta_{\phi} w\right|^{2}+\nabla w \nabla \Delta_{\phi} w+\operatorname{Ric}_{\phi}^{m}(\nabla w, \nabla w) .
\end{aligned}
$$

It follows from $p>1$ that

$$
\begin{aligned}
& \mathscr{L}\left(|\nabla v|^{2}\right) \leq 2 \nabla v \nabla v_{t}-2(p-1) v\left(\frac{1}{m}\left|\Delta_{\phi} v\right|^{2}+\nabla v \nabla \Delta_{\phi} v+\operatorname{Ric}_{\phi}^{m}(\nabla v, \nabla v)\right) \\
&= 2 \nabla v \nabla\left[(p-1) v \Delta_{\phi} v+|\nabla v|^{2}\right] \\
&-2(p-1) v\left(\frac{1}{m}\left|\Delta_{\phi} v\right|^{2}+\nabla v \nabla \Delta_{\phi} v+\operatorname{Ric}_{\phi}^{m}(\nabla v, \nabla v)\right) \\
&=2(p-1)|\nabla v|^{2} \Delta_{\phi} v+2 \nabla v \nabla\left(|\nabla v|^{2}\right) \\
& \quad-\frac{2(p-1)}{m} v\left(\Delta_{\phi} v\right)^{2}-2(p-1) v \operatorname{Ric}_{\phi}^{m}(\nabla v, \nabla v) .
\end{aligned}
$$


Applying this and (2-4) to (2-3) yields

(2-6) $\mathscr{L}\left(\frac{v_{t}}{v}\right)=(p-1) \frac{v_{t}}{v} \Delta_{\phi} v+\frac{2}{v} \nabla v \nabla v_{t}-\frac{v_{t}}{v} \frac{|\nabla v|^{2}}{v}+2(p-1) v \nabla \frac{v_{t}}{v} \nabla \log v$

and

$$
\begin{aligned}
& \mathscr{L}\left(\frac{|\nabla v|^{2}}{v}\right) \leq 2(p-1) \frac{|\nabla v|^{2}}{v} \Delta_{\phi} v+\frac{2}{v} \nabla v \nabla\left(|\nabla v|^{2}\right) \\
& -\frac{2(p-1)}{m}\left(\Delta_{\phi} v\right)^{2}-2(p-1) \operatorname{Ric}_{\phi}^{m}(\nabla v, \nabla v)-\frac{|\nabla v|^{4}}{v^{2}}+2(p-1) v \nabla \frac{|\nabla v|^{2}}{v} \nabla \log v,
\end{aligned}
$$

and hence

$$
\begin{aligned}
& \mathscr{L}(F)=\mathscr{L}(\left.\frac{|\nabla v|^{2}}{v}\right)-\alpha \mathscr{L}\left(\frac{v_{t}}{v}\right)-\alpha^{\prime} \frac{v_{t}}{v}-\varphi^{\prime} \\
& \leq 2(p-1) \frac{|\nabla v|^{2}}{v} \Delta_{\phi} v+\frac{2}{v} \nabla v \nabla\left(|\nabla v|^{2}\right)-\frac{2(p-1)}{m}\left(\Delta_{\phi} v\right)^{2} \\
&-2(p-1) \operatorname{Ric}_{\phi}^{m}(\nabla v, \nabla v)-\frac{|\nabla v|^{4}}{v^{2}}+2(p-1) v \nabla \frac{|\nabla v|^{2}}{v} \nabla \log v \\
&-\alpha(p-1) \frac{v_{t}}{v} \Delta_{\phi} v-\alpha \frac{2}{v} \nabla v \nabla v_{t}+\alpha \frac{v_{t}}{v} \frac{|\nabla v|^{2}}{v} \\
&-2 \alpha(p-1) v \nabla \frac{v_{t}}{v} \nabla \log v-\alpha^{\prime} \frac{v_{t}}{v}-\varphi^{\prime} .
\end{aligned}
$$

Noticing that

$$
2(p-1) v \nabla \frac{|\nabla v|^{2}}{v} \nabla \log v-2 \alpha(p-1) v \nabla \frac{v_{t}}{v} \nabla \log v=2(p-1) \nabla v \nabla F
$$

and

$$
\frac{2}{v} \nabla v \nabla\left(|\nabla v|^{2}\right)-\alpha \frac{2}{v} \nabla v \nabla v_{t}=\frac{2}{v} \nabla v \nabla[(F+\varphi) v]=2(F+\varphi) \frac{|\nabla v|^{2}}{v}+2 \nabla v \nabla F,
$$

we obtain

$$
\begin{aligned}
2(p-1) v\left(\nabla \frac{|\nabla v|^{2}}{v}-\alpha \nabla \frac{v_{t}}{v}\right) \nabla \log v & +\frac{2}{v} \nabla v \nabla\left(|\nabla v|^{2}\right)-\alpha \frac{2}{v} \nabla v \nabla v_{t} \\
& =2 p \nabla v \nabla F+2(F+\varphi) \frac{|\nabla v|^{2}}{v} \\
& =2 p \nabla v \nabla F+2\left(\frac{|\nabla v|^{2}}{v}-\alpha \frac{v_{t}}{v}\right) \frac{|\nabla v|^{2}}{v} .
\end{aligned}
$$

On the other hand, using (2-1) again, we have 


$$
\begin{aligned}
2(p-1) & \frac{|\nabla v|^{2}}{v} \Delta_{\phi} v-\frac{|\nabla v|^{4}}{v^{2}}-\alpha(p-1) \frac{v_{t}}{v} \Delta_{\phi} v+\alpha \frac{v_{t}}{v} \frac{|\nabla v|^{2}}{v} \\
= & 2 \frac{|\nabla v|^{2}}{v}\left(\frac{v_{t}}{v}-\frac{|\nabla v|^{2}}{v}\right)-\frac{|\nabla v|^{4}}{v^{2}}-\alpha \frac{v_{t}}{v}\left(\frac{v_{t}}{v}-\frac{|\nabla v|^{2}}{v}\right)+\alpha \frac{v_{t}}{v} \frac{|\nabla v|^{2}}{v} \\
= & (2 \alpha+2) \frac{v_{t}}{v} \frac{|\nabla v|^{2}}{v}-3 \frac{|\nabla v|^{4}}{v^{2}}-\alpha\left(\frac{v_{t}}{v}\right)^{2} .
\end{aligned}
$$

Combining (2-8) with (2-9) gives

(2-10) $2(p-1) v \nabla \frac{|\nabla v|^{2}}{v} \nabla \log v-2 \alpha(p-1) v \nabla \frac{v_{t}}{v} \nabla \log v+\frac{2}{v} \nabla v \nabla\left(|\nabla v|^{2}\right)$

$$
\begin{array}{r}
-\alpha \frac{2}{v} \nabla v \nabla v_{t}+2(p-1) \frac{|\nabla v|^{2}}{v} \Delta_{\phi} v-\frac{|\nabla v|^{4}}{v^{2}}-\alpha(p-1) \frac{v_{t}}{v} \Delta_{\phi} v+\alpha \frac{v_{t}}{v} \frac{|\nabla v|^{2}}{v} \\
=2 p \nabla v \nabla F-\left(\frac{v_{t}}{v}-\frac{|\nabla v|^{2}}{v}\right)^{2}+(1-\alpha)\left(\frac{v_{t}}{v}\right)^{2} \\
=2 p \nabla v \nabla F-\left[(p-1) \Delta_{\phi} v\right]^{2}+(1-\alpha)\left(\frac{v_{t}}{v}\right)^{2} .
\end{array}
$$

Putting (2-10) into (2-7) yields

$$
\begin{array}{r}
\mathscr{L}(F) \leq-\frac{2(p-1)}{m}\left(\Delta_{\phi} v\right)^{2}-2(p-1) \operatorname{Ric}_{\phi}^{m}(\nabla v, \nabla v)+2 p \nabla v \nabla F \\
-\left[(p-1) \Delta_{\phi} v\right]^{2}+(1-\alpha)\left(\frac{v_{t}}{v}\right)^{2}-\alpha^{\prime} \frac{v_{t}}{v}-\varphi^{\prime} \\
=-\frac{1}{\tilde{a}}\left[(p-1) \Delta_{\phi} v\right]^{2}-2(p-1) \operatorname{Ric}_{\phi}^{m}(\nabla v, \nabla v)+2 p \nabla v \nabla F \\
+(1-\alpha)\left(\frac{v_{t}}{v}\right)^{2}-\alpha^{\prime} \frac{v_{t}}{v}-\varphi^{\prime},
\end{array}
$$

which completes the proof of (1) in Lemma 2.1.

Proof of Theorem 1.1. Let $\xi$ be a cut-off function such that $\xi(r)=1$ for $r \leq 1$, $\xi(r)=0$ for $r \geq 2,0 \leq \xi(r) \leq 1$, and

$$
0 \geq \xi^{\prime}(r) \geq-c_{1} \xi^{1 / 2}(r), \quad \xi^{\prime \prime}(r) \geq-c_{2},
$$

for positive constants $c_{1}$ and $c_{2}$. With $\rho(x)$ the distance between $x$ and $p$ in $M^{n}$, let

$$
\psi(x)=\xi\left(\frac{\rho(x)}{R}\right) .
$$

Making use of an argument of Calabi [1958] (see also [Cheng and Yau 1975]), we can assume without loss of generality that the function $\psi$ is smooth in $B_{p}(2 R)$. Then we have

$$
\frac{|\nabla \psi|^{2}}{\psi} \leq \frac{C}{R^{2}}
$$


By the comparison theorem with respect to the Witten Laplacian (see [Li 2005, p. 1324])

$$
\Delta_{\phi} \rho \geq \sqrt{(m-1) K} \operatorname{coth}\left(\sqrt{\left.\frac{K}{m-1} \rho\right)}\right.
$$

we have

$$
\Delta_{\phi} \psi=\frac{\xi^{\prime} \Delta_{\phi} \rho}{R}+\frac{\xi^{\prime \prime}|\nabla \rho|^{2}}{R^{2}} \geq-\frac{C(m)}{R^{2}}(1+\sqrt{K} R \operatorname{coth}(\sqrt{K} R)) .
$$

Define $\tilde{F}=|\nabla v|^{2} / v-\alpha v_{t} / v$, where $\alpha>1$ is a constant. Under the assumption that $\operatorname{Ric}_{\phi}^{m} \geq-K$, Lemma 2.1(1) shows that

$$
\begin{aligned}
\mathscr{L}(\tilde{F}) & \leq-\frac{1}{\tilde{a}}\left[(p-1) \Delta_{\phi} v\right]^{2}+2(p-1) K|\nabla v|^{2}+2 p \nabla v \nabla \tilde{F} \\
& \leq-\frac{1}{\tilde{a}}\left[(p-1) \Delta_{\phi} v\right]^{2}+2 M K \frac{|\nabla v|^{2}}{v}+2 p \nabla v \nabla \tilde{F} .
\end{aligned}
$$

Set $G=t \psi \widetilde{F}$. Next we will apply the maximum principle to $G$ on $B_{p}(2 R) \times[0, T]$. Assume $G$ achieves its maximum at the point $\left(x_{0}, s\right) \in B_{p}(2 R) \times[0, T]$ and assume $G\left(x_{0}, s\right)>0$ (otherwise the proof is trivial), which implies $s>0$. Then, at the point $\left(x_{0}, s\right)$, we have

$$
\mathscr{L}(G) \geq 0, \quad \nabla \widetilde{F}=-\frac{\tilde{F}}{\psi} \nabla \psi,
$$

and, by use of (2-13), we have

$(2-14) \quad 0 \leq \mathscr{L}(G)$

$$
\begin{aligned}
& =s \psi \mathscr{L}(\tilde{F})-s(p-1) v \widetilde{F} \Delta_{\phi} \psi-2 s(p-1) v \nabla \tilde{F} \nabla \psi+\psi \tilde{F} \\
& =s \psi \mathscr{L}(\tilde{F})-(p-1) v \frac{\Delta_{\phi} \psi}{\psi} G+2(p-1) v \frac{|\nabla \psi|^{2}}{\psi^{2}} G+\frac{G}{s} \\
& \leq s \psi\left(-\frac{1}{\tilde{a}}\left[(p-1) \Delta_{\phi} v\right]^{2}+2 M K \frac{|\nabla v|^{2}}{v}+2 p \nabla v \nabla \tilde{F}\right) \\
& \quad-(p-1) v \frac{\Delta_{\phi} \psi}{\psi} G+2(p-1) v \frac{|\nabla \psi|^{2}}{\psi^{2}} G+\frac{G}{s} \\
& \leq-\frac{s \psi}{\tilde{a}}\left[(p-1) \Delta_{\phi} v\right]^{2}+2 s \psi M K \frac{|\nabla v|^{2}}{v}+2 \frac{p}{\sqrt{p-1}} \sqrt{M} G \frac{|\nabla v||\nabla \psi|}{\sqrt{v}} \frac{\mid \nabla \psi}{\psi} \\
& \quad-(p-1) v \frac{\Delta_{\phi} \psi}{\psi} G+2(p-1) v \frac{|\nabla \psi|^{2}}{\psi^{2}} G+\frac{G}{s} .
\end{aligned}
$$

Applying

$$
\left[(p-1) \Delta_{\phi} v\right]^{2}=\frac{1}{\alpha^{2}} \widetilde{F}^{2}+\frac{2(\alpha-1)}{\alpha^{2}} \widetilde{F} \frac{|\nabla v|^{2}}{v}+\left(\frac{\alpha-1}{\alpha}\right)^{2} \frac{|\nabla v|^{4}}{v^{2}}
$$


to (2-14), we obtain

$$
\begin{aligned}
0 \leq-\frac{1}{\tilde{a} s \alpha^{2}} G^{2}-\frac{2(\alpha-1) \psi}{\tilde{a} \alpha^{2}} & G \frac{|\nabla v|^{2}}{v}-\frac{s \psi^{2}}{\tilde{a}}\left(\frac{\alpha-1}{\alpha}\right)^{2} \frac{|\nabla v|^{4}}{v^{2}} \\
+ & 2 s \psi^{2} M K \frac{|\nabla v|^{2}}{v}+2 \frac{p}{\sqrt{p-1}} \sqrt{M \psi} G \frac{|\nabla v|}{\sqrt{v}} \frac{|\nabla \psi|}{\sqrt{\psi}} \\
& -(p-1) v\left(\Delta_{\phi} \psi\right) G+2(p-1) v \frac{|\nabla \psi|^{2}}{\psi} G+\frac{\psi G}{s} .
\end{aligned}
$$

Since $-A x^{2}+B x \leq \frac{B^{2}}{4 A}$, we have

$$
-\frac{s \psi^{2}}{\tilde{a}}\left(\frac{\alpha-1}{\alpha}\right)^{2} \frac{|\nabla v|^{4}}{v^{2}}+2 s \psi^{2} M K \frac{|\nabla v|^{2}}{v} \leq \frac{\tilde{a} \alpha^{2} s \psi^{2} M^{2} K^{2}}{(\alpha-1)^{2}}
$$

and

$$
-\frac{2(\alpha-1) \psi}{\tilde{a} \alpha^{2}} G \frac{|\nabla v|^{2}}{v}+\frac{2 p}{\sqrt{p-1}} \sqrt{M \psi} G \frac{|\nabla v|}{\sqrt{v}} \frac{|\nabla \psi|}{\sqrt{\psi}} \leq \frac{\tilde{a} \alpha^{2} p^{2} M}{2(p-1)(\alpha-1)} \frac{|\nabla \psi|^{2}}{\psi} G .
$$

We now set

$$
P(K, R)=1+\sqrt{K} R \operatorname{coth}(\sqrt{K} R) .
$$

From (2-15) we obtain

$$
\begin{aligned}
& 0 \leq-\frac{1}{\tilde{a} s \alpha^{2}} G^{2}+\frac{\tilde{a} \alpha^{2} s \psi^{2} M^{2} K^{2}}{(\alpha-1)^{2}}+ \frac{\tilde{a} \alpha^{2} p^{2} M}{2(p-1)(\alpha-1)} \frac{|\nabla \psi|^{2}}{\psi} G \\
&-(p-1) v(L \psi) G+2(p-1) v \frac{|\nabla \psi|^{2}}{\psi} G+\frac{\psi G}{s} \\
& \leq-\frac{1}{\tilde{a} s \alpha^{2}} G^{2}+\left(\frac{\tilde{a} \alpha^{2} p^{2} M}{2(p-1)(\alpha-1)} \frac{C}{R^{2}}+M \frac{C(m)}{R^{2}} P(K, R)+\frac{\psi}{s}\right) G \\
&+\frac{\tilde{a} \alpha^{2} s \psi^{2} M^{2} K^{2}}{(\alpha-1)^{2}} .
\end{aligned}
$$

Solving this quadratic inequality for $G$ yields

$$
\begin{aligned}
G \leq & \frac{\tilde{a} s \alpha^{2}}{2}\left\{\frac{\tilde{a} \alpha^{2} p^{2} M}{2(p-1)(\alpha-1)} \frac{C}{R^{2}}+M \frac{C(m)}{R^{2}} P(K, R)+\frac{\psi}{s}\right. \\
& \left.+\left[\left(\frac{\tilde{a} \alpha^{2} p^{2} M}{2(p-1)(\alpha-1)} \frac{C}{R^{2}}+M \frac{C(m)}{R^{2}} P(K, R)+\frac{\psi}{s}\right)^{2}+\frac{4 \psi^{2} M^{2} K^{2}}{(\alpha-1)^{2}}\right]^{\frac{1}{2}}\right\} \\
\leq & \tilde{a} s \alpha^{2}\left\{\frac{\tilde{a} \alpha^{2} p^{2} M}{2(p-1)(\alpha-1)} \frac{C}{R^{2}}+M \frac{C(m)}{R^{2}} P(K, R)+\frac{\psi}{s}+\frac{\psi M K}{\alpha-1}\right\} .
\end{aligned}
$$


Hence we have

$G(x, T) \leq G\left(x_{0}, s\right)$

$$
\leq \tilde{a} T \alpha^{2} \frac{C(m)}{R^{2}}\left(\frac{\alpha^{2}}{(p-1)(\alpha-1)} \tilde{a} p^{2}+P(K, R)\right) M+\frac{\alpha^{2}}{\alpha-1} \tilde{a} T M K+\tilde{a} \alpha^{2} .
$$

This implies that, for all $x \in B_{p}(R)$,

$$
F(x, T) \leq \tilde{a} \alpha^{2} M \frac{C(m)}{R^{2}}\left(\frac{\alpha^{2}}{\alpha-1} \frac{\tilde{a} p^{2}}{p-1}+P(K, R)\right)+\frac{\alpha^{2}}{\alpha-1} \tilde{a} M K+\frac{\tilde{a} \alpha^{2}}{T} .
$$

Since $T$ is arbitrary, we complete the proof of Theorem 1.1.

Proof of Corollary 1.2. Along the lines of Li and Yau, we will establish a Harnack inequality from a general estimate

$$
\frac{|\nabla v|^{2}}{v}-\alpha(t) \frac{v_{t}}{v}-\varphi(t) \leq 0
$$

Rewrite (2-18) as

$$
-\frac{v_{t}}{v} \leq \frac{1}{\alpha(t)}\left(\varphi(t)-\frac{|\nabla v|^{2}}{v}\right) .
$$

Let $f=\log v$. Then we have

$$
-f_{t}=-\frac{v_{t}}{v} \leq \frac{1}{\alpha(t)}\left(\varphi(t)-\frac{|\nabla v|^{2}}{v}\right) \leq \frac{1}{\alpha(t)}\left(\varphi(t)-\widetilde{M}|\nabla f|^{2}\right) .
$$

Let $\gamma$ be a shortest geodesic joining $x_{1}$ and $x_{2}$, and set $\gamma:\left[t_{1}, t_{2}\right] \rightarrow M^{n}, \gamma\left(t_{1}\right)=x_{1}$, $\gamma\left(t_{2}\right)=x_{2}$. Define a curve $\zeta$ in $M^{n} \times(0, \infty), \zeta:\left[t_{1}, t_{2}\right] \rightarrow M^{n} \times(0, \infty)$ by $\zeta(t)=(\gamma(t), t)$. Then $\zeta\left(t_{1}\right)=\left(x_{1}, t_{1}\right)$ and $\zeta\left(t_{2}\right)=\left(x_{2}, t_{2}\right)$. Set $\rho=d\left(x_{1}, x_{2}\right)$. Then $|\dot{\gamma}|=\rho /\left(t_{2}-t_{1}\right)$ and

$$
\begin{aligned}
f\left(x_{1}, t_{1}\right)-f\left(x_{2}, t_{2}\right) & =\int_{t_{2}}^{t_{1}} \frac{d}{d t} f(\zeta(t)) d t=\int_{t_{2}}^{t_{1}}\left(\langle\dot{\gamma}, \nabla f\rangle+f_{t}\right) d t \\
& =\int_{t_{1}}^{t_{2}}\left(-\langle\dot{\gamma}, \nabla f\rangle+\left(-f_{t}\right)\right) d t \\
& \leq \int_{t_{1}}^{t_{2}}\left(|\dot{\gamma}||\nabla f|+\frac{1}{\alpha(t)}\left(\varphi(t)-\widetilde{M}|\nabla f|^{2}\right)\right) d t \\
& =\int_{t_{1}}^{t_{2}}\left(-\frac{\widetilde{M}}{\alpha(t)}|\nabla f|^{2}+|\dot{\gamma}||\nabla f|\right) d t+\int_{t_{1}}^{t_{2}} \frac{\varphi(t)}{\alpha(t)} d t \\
& \leq \frac{\rho^{2}}{4 \widetilde{M}\left(t_{2}-t_{1}\right)^{2}} \int_{t_{1}}^{t_{2}} \alpha(t) d t+\int_{t_{1}}^{t_{2}} \frac{\varphi(t)}{\alpha(t)} d t,
\end{aligned}
$$

where in the last inequality we used $-A x^{2}+B x \leq \frac{B^{2}}{4 A}$ and $|\dot{\gamma}|=\rho /\left(t_{2}-t_{1}\right)$. 
Let $\alpha>1$ be a constant and set $\varphi=\frac{\alpha^{2}}{(\alpha-1)} \tilde{a} M K+\frac{\tilde{a} \alpha^{2}}{t}$. We have from (2-19)

$$
\begin{aligned}
f\left(x_{1}, t_{1}\right)-f\left(x_{2}, t_{2}\right) & \leq \int_{t_{1}}^{t_{2}}\left(\frac{\alpha \rho^{2}}{4 \widetilde{M}\left(t_{2}-t_{1}\right)^{2}}+\frac{\alpha}{\alpha-1} \tilde{a} M K+\frac{\tilde{a} \alpha}{t}\right) d t \\
& =\frac{\alpha \rho^{2}}{4 \widetilde{M}\left(t_{2}-t_{1}\right)}+\frac{\alpha}{\alpha-1} \tilde{a} M K\left(t_{2}-t_{1}\right)+\tilde{a} \alpha \log \frac{t_{2}}{t_{1}} .
\end{aligned}
$$

Therefore, we arrive at

$$
v\left(x_{1}, t_{1}\right) \leq v\left(x_{2}, t_{2}\right)\left(\frac{t_{2}}{t_{1}}\right)^{\tilde{a} \alpha} \exp \left(\frac{\alpha \rho^{2}}{4 \widetilde{M}\left(t_{2}-t_{1}\right)}+\frac{\alpha}{\alpha-1} \tilde{a} M K\left(t_{2}-t_{1}\right)\right) .
$$

Proof of Theorem 1.3. When $p \in(0,1)$, we have $v<0$, and from Lemma 2.1(2) $\mathscr{L}(-\tilde{F}) \leq \frac{1}{\tilde{a}}\left[(p-1) \Delta_{\phi} v\right]^{2}+2(p-1) \operatorname{Ric}_{\phi}^{m}(\nabla v, \nabla v)+2 p \nabla v \nabla(-\tilde{F})-(1-\alpha)\left(\frac{v_{t}}{v}\right)^{2}$,

which implies

$(2-21) \mathscr{L}(-\tilde{F}) \leq \frac{1}{\tilde{a}}\left[(p-1) \Delta_{\phi} v\right]^{2}+2 M K \frac{|\nabla v|^{2}}{-v}+2 p \nabla v \nabla(-\tilde{F})-(1-\alpha)\left(\frac{v_{t}}{v}\right)^{2}$.

Define $G=t \psi(-\widetilde{F})$. We'll apply the maximum principle to $G$ on $B_{p}(2 R) \times[0, T]$. Assume $G$ achieves its maximum at the point $\left(x_{0}, s\right) \in B_{p}(2 R) \times[0, T]$ and assume $G\left(x_{0}, s\right)>0$ (otherwise the proof is trivial), which implies $s>0$. Then, at the point $\left(x_{0}, s\right)$, we have

$$
\mathscr{L}(G) \geq 0, \quad \nabla(-\widetilde{F})=-\frac{-\widetilde{F}}{\psi} \nabla \psi
$$

and, by use of (2-21), we have

$$
\begin{gathered}
0 \leq \mathscr{L}(G)=s \psi \mathscr{L}(-\tilde{F})-(p-1) v \frac{\Delta_{\phi} \psi}{\psi} G+2(p-1) v \frac{|\nabla \psi|^{2}}{\psi^{2}} G+\frac{G}{s} \\
\leq s \psi\left(\frac{1}{\tilde{a}}\left[(p-1) \Delta_{\phi} v\right]^{2}+2 M K \frac{|\nabla v|^{2}}{-v}+2 p \nabla v \nabla(-\tilde{F})\right) \\
\quad-(p-1) v \frac{\Delta_{\phi} \psi}{\psi} G+2(p-1) v \frac{|\nabla \psi|^{2}}{\psi^{2}} G+\frac{G}{s}-(1-\alpha) s \psi\left(\frac{v_{t}}{v}\right)^{2} \\
\leq \frac{s \psi}{\tilde{a}}\left[(p-1) \Delta_{\phi} v\right]^{2}+2 s \varphi M K \frac{|\nabla v|^{2}}{-v} \\
+2 \frac{p}{\sqrt{1-p}} \sqrt{M} G \frac{|\nabla v|}{\sqrt{-v}} \frac{|\nabla \psi|}{\psi}-(p-1) v \frac{\Delta_{\phi} \psi}{\psi} G \\
+2(p-1) v \frac{|\nabla \psi|^{2}}{\psi \psi^{2}} G+\frac{G}{s}-(1-\alpha) s \psi\left(\frac{v_{t}}{v}\right)^{2} .
\end{gathered}
$$


Applying the equalities

$$
\left[(p-1) \Delta_{\phi} v\right]^{2}=\frac{1}{\alpha^{2}} \tilde{F}^{2}+\frac{2(\alpha-1)}{\alpha^{2}} \tilde{F} \frac{|\nabla v|^{2}}{v}+\left(\frac{\alpha-1}{\alpha}\right)^{2} \frac{|\nabla v|^{4}}{v^{2}}
$$

and

$$
\left(\frac{v_{t}}{v}\right)^{2}=\frac{1}{\alpha^{2}}\left(-\tilde{F}+\frac{|\nabla v|^{2}}{v}\right)^{2}=\frac{1}{\alpha^{2}}(-\tilde{F})^{2}+\frac{2}{\alpha^{2}}(-\widetilde{F}) \frac{|\nabla v|^{2}}{v}+\frac{1}{\alpha^{2}} \frac{|\nabla v|^{4}}{v^{2}}
$$

to $(2-22)$, we obtain

$$
\begin{aligned}
0 \leq & \frac{1}{\tilde{a} s \alpha^{2}}\left((1-\tilde{a}(1-\alpha)) G^{2}-2(1-\tilde{a})(1-\alpha) s \psi G \frac{|\nabla v|^{2}}{-v}\right. \\
& \left.+s^{2} \psi^{2}(1-\alpha)(1-\alpha-\tilde{a}) \frac{|\nabla v|^{4}}{v^{2}}\right) \\
& +2 s \psi^{2} M K \frac{|\nabla v|^{2}}{-v}+2 \frac{p}{\sqrt{1-p}} \sqrt{M \psi} G \frac{|\nabla v|}{\sqrt{-v}} \frac{|\nabla \psi|}{\sqrt{\psi}} \\
& -(p-1) v\left(\Delta_{\phi} \psi\right) G+2(p-1) v \frac{|\nabla \psi|^{2}}{\psi} G+\frac{\psi G}{s} .
\end{aligned}
$$

Next we employ a method similar to that in [Lu et al. 2009, Theorem 4.1]. Since $p \in(1-2 / m, 1)$, we have $\tilde{a}<0$. Thus we have, for any positive constants $\varepsilon_{1}, \varepsilon_{2}$,

$$
2 s \psi^{2} M K \frac{|\nabla v|^{2}}{-v} \leq-\varepsilon_{1} \frac{s^{2} \psi^{2}}{\tilde{a} s \alpha^{2}}(1-\alpha)(1-\alpha-\tilde{a}) \frac{|\nabla v|^{4}}{v^{2}}-\frac{1}{\varepsilon_{1}} \frac{\tilde{a} s \alpha^{2}(p-1)^{2} \psi^{2} M^{2} K^{2}}{(1-\alpha)(1-\alpha-\tilde{a})}
$$

and

$$
\begin{aligned}
2 \frac{p}{\sqrt{1-p}} & \sqrt{M \psi} G \frac{|\nabla v|}{\sqrt{-v}} \frac{|\nabla \psi|}{\sqrt{\psi}} \\
& \leq-\varepsilon_{2} \frac{2}{\tilde{a} s \alpha^{2}}(1-\tilde{a})(1-\alpha) s \psi G \frac{|\nabla v|^{2}}{-v}-\frac{\tilde{a} \alpha^{2} p^{2} M}{2 \varepsilon_{2}(1-\tilde{a})(1-\alpha)(1-p)} \frac{|\nabla \psi|^{2}}{\psi} G .
\end{aligned}
$$

Hence we get from (2-23) that

$$
\begin{aligned}
0 \leq & -\frac{1}{\tilde{a} s \alpha^{2}}\left(-(1-\tilde{a}(1-\alpha)) G^{2}+2\left(1+\varepsilon_{2}\right)(1-\tilde{a})(1-\alpha) s \psi G \frac{|\nabla v|^{2}}{-v}\right. \\
& \left.-\left(1-\varepsilon_{1}\right) s^{2} \psi^{2}(1-\alpha)(1-\alpha-\tilde{a}) \frac{|\nabla v|^{4}}{v^{2}}\right) \\
& -\frac{1}{\varepsilon_{1}} \frac{a s \alpha^{2} \psi^{2} M^{2} K^{2}}{(1-\alpha)(1-\alpha-\tilde{a})}-\frac{\tilde{a} \alpha^{2} p^{2} M}{2 \varepsilon_{2}(1-\tilde{a})(1-\alpha)(1-p)} \frac{|\nabla \psi|^{2}}{\psi} G \\
& -(p-1) v\left(\Delta_{\phi} \psi\right) G+2(p-1) v \frac{|\nabla \psi|^{2}}{\psi} G+\frac{\psi G}{s},
\end{aligned}
$$

which can be rewritten as 
$(2-24)$

$$
\begin{aligned}
& 0 \leq \frac{1}{\tilde{a} s \alpha^{2}}(1-\tilde{a}(1-\alpha)\left.-\frac{\left(1+\varepsilon_{2}\right)^{2}(1-\tilde{a})^{2}(1-\alpha)}{\left(1-\varepsilon_{1}\right)(1-\alpha-\tilde{a})}\right) G^{2} \\
&-\frac{1}{\varepsilon_{1}} \frac{\tilde{a} s \alpha^{2} \psi^{2} M^{2} K^{2}}{(1-\alpha)(1-\alpha-\tilde{a})}-\frac{\tilde{a} \alpha^{2} p^{2} M}{2 \varepsilon_{2}(1-\tilde{a})(1-\alpha)(1-p)} \frac{|\nabla \psi|^{2}}{\psi} G \\
& \quad-(p-1) v\left(\Delta_{\phi} \psi\right) G+2(p-1) v \frac{|\nabla \psi|^{2}}{\psi} G+\frac{\psi G}{s} .
\end{aligned}
$$

Taking $\varepsilon_{1}, \varepsilon_{2}$ such that

$$
1-\tilde{a}(1-\alpha)-\frac{\left(1+\varepsilon_{2}\right)^{2}(1-\tilde{a})^{2}(1-\alpha)}{\left(1-\varepsilon_{1}\right)(1-\alpha-\tilde{a})}=: A\left(\varepsilon_{1}, \varepsilon_{2}\right)>0,
$$

we obtain from (2-24), with $P(K, R)$ as in (2-16),

$$
\begin{aligned}
0 \leq-\frac{1}{(-\tilde{a}) s \alpha^{2}} A\left(\varepsilon_{1}, \varepsilon_{2}\right) G^{2} & \\
+\left(\frac{(-\tilde{a}) \alpha^{2} p^{2} M}{2 \varepsilon_{2}(1-\tilde{a})(1-\alpha)(1-p)} \frac{C}{R^{2}}+M \frac{C(m)}{R^{2}} P(K, R)\right. & \left.+\frac{\psi}{s}\right) G \\
& +\frac{(-\tilde{a}) s \alpha^{2} \psi^{2} M^{2} K^{2}}{\varepsilon_{1}(1-\alpha)(1-\alpha-\tilde{a})} .
\end{aligned}
$$

Solving this quadratic inequality for $G$ yields

$$
\begin{array}{r}
G \leq \frac{(-\tilde{a}) s \alpha^{2}}{A\left(\varepsilon_{1}, \varepsilon_{2}\right)}\left(\frac{(-\tilde{a}) \alpha^{2} p^{2} M}{2 \varepsilon_{2}(1-\tilde{a})(1-\alpha)(1-p)} \frac{C}{R^{2}}+M \frac{C(m)}{R^{2}} P(K, R)\right. \\
\left.+\frac{\psi}{s}+\frac{\psi M K}{\sqrt{\varepsilon_{1}(1-\alpha)(1-\alpha-\tilde{a})}} \sqrt{A\left(\varepsilon_{1}, \varepsilon_{2}\right)}\right) .
\end{array}
$$

Hence we have

$$
\begin{aligned}
G(x, T) \leq & G\left(x_{0}, s\right) \\
\leq & \frac{(-\tilde{a}) T \alpha^{2} M}{A\left(\varepsilon_{1}, \varepsilon_{2}\right)} \frac{C(m)}{R^{2}}\left(\frac{(-\tilde{a}) \alpha^{2} p^{2}}{2 \varepsilon_{2}(1-\tilde{a})(1-\alpha)(1-p)}+P(K, R)\right) \\
& +\frac{(-\tilde{a}) T \alpha^{2} M K}{\sqrt{\varepsilon_{1}(1-\alpha)(1-\alpha-\tilde{a}) A\left(\varepsilon_{1}, \varepsilon_{2}\right)}}+\frac{(-\tilde{a}) \alpha^{2}}{A\left(\varepsilon_{1}, \varepsilon_{2}\right)},
\end{aligned}
$$

and, for $x \in B_{p}(R)$,

$$
\begin{aligned}
-F(x, t) \leq \frac{(-\tilde{a}) \alpha^{2} M}{A\left(\varepsilon_{1}, \varepsilon_{2}\right)} \frac{C(m)}{R^{2}}\left(\frac{(-\tilde{a}) \alpha^{2} p^{2}}{2 \varepsilon_{2}(1-\tilde{a})(1-\alpha)(1-p)}+P(K, R)\right) & \\
& +\frac{(-\tilde{a}) \alpha^{2} M K}{\sqrt{\varepsilon_{1}(1-\alpha)(1-\alpha-\tilde{a}) A\left(\varepsilon_{1}, \varepsilon_{2}\right)}}+\frac{(-\tilde{a}) \alpha^{2}}{A\left(\varepsilon_{1}, \varepsilon_{2}\right) t} .
\end{aligned}
$$

This completes the proof of Theorem 1.3. 
Proof of Corollary 1.4. Choosing $f=\log (-v)$ and $\varphi(t)=-\frac{\tilde{a}}{t}$, we get from (2-19) $f\left(x_{2}, t_{2}\right)-f\left(x_{1}, t_{1}\right) \leq \int_{t_{1}}^{t_{2}}\left(\frac{\rho^{2}}{4 \widetilde{M}\left(t_{2}-t_{1}\right)^{2}}-\frac{\tilde{a}}{t}\right) d t=\frac{\rho^{2}}{4 \widetilde{M}\left(t_{2}-t_{1}\right)}-\tilde{a} \log \frac{t_{2}}{t_{1}}$.

\section{Proof of Theorem 1.6}

Proof. Define $\bar{F}=\frac{|\nabla v|^{2}}{v}-\alpha \frac{v_{t}}{v}$, where $\alpha \in(0,1)$ is constant. Lemma 2.1(2) shows that

$$
\begin{array}{r}
\mathscr{L}(-\bar{F}) \leq \frac{1}{\tilde{a}}\left[(p-1) \Delta_{\phi} v\right]^{2}+2 M K \frac{|\nabla v|^{2}}{-v}+2 p \nabla v \nabla(-\bar{F})-(1-\alpha)\left(\frac{v_{t}}{v}\right)^{2} \\
=\frac{1}{\tilde{a} \alpha^{2}}\left(-\bar{F}-(1-\alpha) \frac{|\nabla v|^{2}}{-v}\right)^{2}+2 M K \frac{|\nabla v|^{2}}{-v}+2 p \nabla v \nabla(-\bar{F}) \\
-\frac{1-\alpha}{\alpha^{2}}\left(-\bar{F}-\frac{|\nabla v|^{2}}{-v}\right)^{2} .
\end{array}
$$

Let $G=t \psi(-\bar{F})$. We apply the maximum principle to $G$ on $B_{p}(2 R) \times[0, T]$ and assume that $G$ achieves its maximum at the point $\left(x_{0}, s\right) \in B_{p}(2 R) \times[0, T]$ with $G\left(x_{0}, s\right)>0$ (otherwise the proof is trivial). At the point $\left(x_{0}, s\right)$, we have

$$
\mathscr{L}(G) \geq 0, \quad \nabla(-\bar{F})=-\frac{-\bar{F}}{\psi} \nabla \psi,
$$

and, by use of (3-1), we get

$$
\begin{aligned}
0 \leq \mathscr{L}(G)= & s \psi \mathscr{L}(-\bar{F})-(p-1) v \frac{\Delta_{\phi} \psi}{\psi} G+2(p-1) v \frac{|\nabla \psi|^{2}}{\psi^{2}} G+\frac{G}{s} \\
\leq \frac{s \psi}{\tilde{a} \alpha^{2}}\left(-\bar{F}-(1-\alpha) \frac{|\nabla v|^{2}}{-v}\right)^{2}+ & 2 s \varphi M K \frac{|\nabla v|^{2}}{-v} \\
& +2 \frac{p}{\sqrt{1-p}} \sqrt{M} G \frac{|\nabla v|}{\sqrt{-v}} \frac{|\nabla \psi|}{\psi}-\frac{1-\alpha}{\alpha^{2}} s \psi\left(-\bar{F}-\frac{|\nabla v|^{2}}{-v}\right)^{2} \\
& \quad-(p-1) v \frac{\Delta_{\phi} \psi}{\psi} G+2(p-1) v \frac{|\nabla \psi|^{2}}{\psi^{2}} G+\frac{G}{s} .
\end{aligned}
$$

Let $\frac{|\nabla v|^{2}}{-v}=\mu(-\bar{F})$ at the point $\left(x_{0}, s\right)$. Then we have $\mu \geq 0$ and

$$
\begin{aligned}
0 \leq & \frac{1}{\tilde{a} \alpha^{2} s \psi}[1-(1-\alpha) \mu]^{2} G^{2}+2 \mu M K G+\frac{2 \sqrt{\mu}}{\sqrt{s \psi}} \frac{p}{\sqrt{1-p}} \sqrt{M} G^{3 / 2} \frac{|\nabla \psi|}{\psi} \\
& -\frac{1-\alpha}{\alpha^{2}} \frac{1}{s \psi}(1-\mu)^{2} G^{2}-(p-1) v \frac{\Delta_{\phi} \psi}{\psi} G+2(p-1) v \frac{|\nabla \psi|^{2}}{\psi^{2}} G+\frac{G}{s} .
\end{aligned}
$$


Multiplying both sides of (3-2) by $s \psi / G$ yields

$$
\begin{aligned}
0 \leq \frac{1}{\tilde{a} \alpha^{2}}[1- & (1-\alpha) \mu]^{2} G+2 \mu M K s \psi+2 \sqrt{\mu s} \frac{p}{\sqrt{1-p}} \sqrt{M} \frac{|\nabla \psi|}{\sqrt{\psi G}} \\
& -\frac{1-\alpha}{\alpha^{2}}(1-\mu)^{2} G-(p-1) s v \Delta_{\phi} \psi+2(p-1) s v \frac{|\nabla \psi|^{2}}{\psi}+\psi
\end{aligned}
$$

Introducing

$$
\begin{aligned}
& \tilde{A}=\frac{1}{-\tilde{a} \alpha^{2}}[1-(1-\alpha) \mu]^{2}+\frac{1-\alpha}{\alpha^{2}}(1-\mu)^{2}, \\
& \tilde{B}=\sqrt{\mu s} \frac{p}{\sqrt{1-p}} \sqrt{M} \frac{|\nabla \psi|}{\sqrt{\psi}}, \\
& \tilde{C}=2 \mu M K s \psi+(1-p) s(-v)\left(-\Delta_{\phi} \psi+2 \frac{|\nabla \psi|^{2}}{\psi}\right)+\psi,
\end{aligned}
$$

we write (3-3) as

$$
0 \leq-\tilde{A} G+2 \tilde{B} G^{1 / 2}+\tilde{C}
$$

It is easy to see that

$$
\begin{aligned}
\frac{1}{\tilde{A}} & =\frac{(-\tilde{a}) \alpha^{2}}{[1-(1-\alpha) \mu]^{2}+(-\tilde{a})(1-\alpha)(1-\mu)^{2}} \\
& =\frac{(-\tilde{a}) \alpha^{2}}{1+(-\tilde{a})(1-\alpha)-2(1-\alpha)(1-\tilde{a}) \mu+(1-\alpha)(1-\alpha-\tilde{a}) \mu^{2}} \leq 1-\alpha-\tilde{a}
\end{aligned}
$$

and

$$
\begin{aligned}
\frac{2 \mu}{\tilde{A}} & =\frac{2(-\tilde{a}) \alpha^{2} \mu}{1+(-\tilde{a})(1-\alpha)-2(1-\alpha)(1-\tilde{a}) \mu+(1-\alpha)(1-\alpha-\tilde{a}) \mu^{2}} \\
& \leq \frac{(-\tilde{a}) \alpha^{2}}{\sqrt{[1+(-\tilde{a})(1-\alpha)](1-\alpha)(1-\alpha-\tilde{a})}-(1-\alpha)(1-\tilde{a})} \\
& =\sqrt{[1 /(1-\alpha)+(-\tilde{a})](1-\alpha-\tilde{a})}+(1-\tilde{a}) \\
& \leq \frac{\alpha^{2}}{2(1-\alpha)}+2(1-\tilde{a}),
\end{aligned}
$$

where the last inequality used that $\sqrt{x y} \leq \frac{1}{2}(x+y)$. Hence there exists a constant $C(\tilde{a}, \alpha)$ such that $\sqrt{\mu} / \tilde{A} \leq C(\tilde{a}, \alpha)$. Now, regarding (3-4) as a quadratic inequality in $\sqrt{G}$ gives

$$
\sqrt{G} \leq 2 \tilde{B} / \tilde{A}+\sqrt{\tilde{C} / \tilde{A}}
$$


and therefore

$$
\begin{aligned}
G^{1 / 2} \leq C(\tilde{a}, \alpha) \sqrt{s M} \frac{p}{\sqrt{1-p}} \frac{C}{R}+ & {\left[\left(\frac{\alpha^{2}}{2(1-\alpha)}+2(1-\tilde{a})\right) M K s+1-\alpha-\tilde{a}\right.} \\
& \left.+(1-p)(1-\alpha-\tilde{a}) M s \frac{C(m)}{R^{2}} P(K, R)\right]^{\frac{1}{2}}
\end{aligned}
$$

Hence, for $x \in B_{p}(R)$, we have

$$
\begin{aligned}
-\frac{|\nabla v|^{2}}{v}+\alpha \frac{v_{t}}{v} & \\
\leq\left\{C(\tilde{a}, \alpha) \frac{p}{\sqrt{1-p}} \sqrt{M} \frac{C}{R}+\right. & {\left[\left(\frac{\alpha^{2}}{2(1-\alpha)}+2(1-\tilde{a})\right) M K+\frac{1-\alpha-\tilde{a}}{t}\right.} \\
& \left.\left.+(1-p)(1-\alpha-\tilde{a}) M \frac{C(m)}{R^{2}} P(K, R)\right]^{\frac{1}{2}}\right\}^{2} .
\end{aligned}
$$

This completes the proof of Theorem 1.6.

On the other hand, under the assumption that $\operatorname{Ric}_{\phi}^{m} \geq-K$ and $p>1$, Lemma 2.1(1) shows that

$\mathscr{L}(F)$

$$
\begin{aligned}
& \leq-\frac{1}{\tilde{a}}\left[(p-1) \Delta_{\phi} v\right]^{2}+2(p-1) K|\nabla v|^{2}+2 p \nabla v \nabla F+(1-\alpha)\left(\frac{v_{t}}{v}\right)^{2}-\alpha^{\prime} \frac{v_{t}}{v}-\varphi^{\prime} \\
& \leq-\frac{1}{\tilde{a}}\left[(p-1) \Delta_{\phi} v\right]^{2}+2 M K \frac{|\nabla v|^{2}}{v}+2 p \nabla v \nabla F+(1-\alpha)\left(\frac{v_{t}}{v}\right)^{2}-\alpha^{\prime} \frac{v_{t}}{v}-\varphi^{\prime} .
\end{aligned}
$$

Following the methods in [Huang et al. 2013], we can prove the following results.

Theorem 3.1. Let $\left(M^{n}, g\right)$ be a complete Riemannian manifold with

$$
\operatorname{Ric}_{\phi}^{m}\left(B_{p}(2 R)\right) \geq-K,
$$

where $\operatorname{Ric}_{\phi}^{m}\left(B_{p}(2 R)\right)$ denotes the m-dimensional Bakry-Emery Ricci curvature on the geodesic ball $B_{p}(2 R)$ with radius $2 R$, and $K$ is a nonnegative constant. Let $u$ be a positive solution to the porous medium equation (1-8) with $p>1$. Set

$$
v=\frac{p}{p-1} u^{p-1}, \quad M=(p-1) \max _{B_{p}(2 R) \times[0, T]} v .
$$

Then, for any $\alpha>1$ and with $\tilde{a}$ as in (1-9), we have on $B_{p}(R)$

$$
\begin{aligned}
& \frac{|\nabla v|^{2}}{v}-\alpha \frac{v_{t}}{v} \\
& \quad \leq \tilde{a} \alpha^{2}\left\{\frac{\sqrt{\tilde{a}} \alpha p \sqrt{M}}{\sqrt{p-1} \sqrt{\alpha-1}} \frac{C(m)}{R}+\left(\frac{1}{t}+\frac{M K}{2(\alpha-1)}+M \frac{C(m)}{R^{2}} P(K, R)\right)^{\frac{1}{2}}\right\}^{2} .
\end{aligned}
$$


Taking $R \rightarrow \infty$, we thus obtain the following estimate on $\left(M^{n}, g\right)$ :

$$
\frac{|\nabla v|^{2}}{v}-\alpha \frac{v_{t}}{v} \leq \frac{\alpha^{2}}{2(\alpha-1)} \tilde{a} M K+\frac{\tilde{a} \alpha^{2}}{t} .
$$

Corollary 3.2. Let $\left(M^{n}, g\right)$ be a complete noncompact Riemannian manifold with $\operatorname{Ric}_{\phi}^{m} \geq-K$, where $K$ is a nonnegative constant. Let $u$ be a positive solution to (1-8) with $p>1$. Set

$$
v=\frac{p}{p-1} u^{p-1}, \quad M=(p-1) \sup _{M^{n} \times[0, T]} v, \quad \widetilde{M}=\inf _{M^{n} \times[0, T]} v .
$$

Then, for any $x_{1}, x_{2} \in M^{n}, 0<t_{1}<t_{2}<T, \alpha>1$, we have

$$
v\left(x_{1}, t_{1}\right) \leq v\left(x_{2}, t_{2}\right)\left(\frac{t_{2}}{t_{1}}\right)^{\tilde{a} \alpha} \exp \left(\frac{\alpha \operatorname{dist}^{2}\left(x_{2}, x_{1}\right)}{4 \widetilde{M}\left(t_{2}-t_{1}\right)}+\frac{\alpha}{2(\alpha-1)} \tilde{a} M K\left(t_{2}-t_{1}\right)\right),
$$

where $\operatorname{dist}\left(x_{2}, x_{1}\right)$ is the distance between $x_{1}$ and $x_{2}$.

Theorem 3.3. Let $\left(M^{n}, g\right)$ and $K$ be as in Theorem 3.1. Let $u$ be a positive solution to the porous medium equation (1-8) with $p>1$. Set

$$
v=\frac{p}{p-1} u^{p-1}, \quad M=(p-1) \max _{B_{p}(2 R) \times[0, T]} v .
$$

Then, for any $\alpha>1$, we have on $B_{p}(R)$

$$
\begin{aligned}
\frac{|\nabla v|^{2}}{v} & -\alpha(t) \frac{v_{t}}{v} \\
& \leq \tilde{a} \alpha^{2}(t) M \frac{C(m)}{R^{2}}\left(\frac{p^{2} \tilde{a} \alpha^{2}(t)}{2(p-1)(\alpha(t)-1)}+3+\sqrt{K} R \operatorname{coth}(\sqrt{K} R)\right)+\frac{\tilde{a} \alpha^{2}(t)}{t},
\end{aligned}
$$

where $\alpha(t)=e^{2 M K t}$. Taking $R \rightarrow \infty$, we thus obtain the following estimate on $\left(M^{n}, g\right)$ :

$$
\frac{|\nabla v|^{2}}{v}-\alpha(t) \frac{v_{t}}{v} \leq \frac{\tilde{a} \alpha^{2}(t)}{t} .
$$

Corollary 3.4. Let $\left(M^{n}, g\right)$ be a complete noncompact Riemannian manifold with $\operatorname{Ric}_{\phi}^{m} \geq-K$, where $K$ is a nonnegative constant. Let $u$ be a positive solution to (1-8) with $p>1$. Set

$$
v=\frac{p}{p-1} u^{p-1}, \quad M=(p-1) \sup _{M^{n} \times[0, T]} v, \quad \widetilde{M}=\inf _{M^{n} \times[0, T]} v .
$$

Then, for any $x_{1}, x_{2} \in M^{n}, 0<t_{1}<t_{2}<T, \alpha>1$, we have

$$
v\left(x_{1}, t_{1}\right) \leq v\left(x_{2}, t_{2}\right) \exp \left\{\frac{e^{2 M K t_{2}}-e^{2 M K t_{1}}}{2 M K}\left(\frac{\operatorname{dist}^{2}\left(x_{2}, x_{1}\right)}{4 \widetilde{M}\left(t_{2}-t_{1}\right)^{2}}+\frac{\tilde{a}}{t_{1}}\right)\right\},
$$

where $\operatorname{dist}\left(x_{2}, x_{1}\right)$ is the distance between $x_{1}$ and $x_{2}$. 
Remark 3.5. Theorems 3.1 and 3.3 reduce to Theorems 1.1 and 1.2 from [Huang et al. 2013], respectively, by letting $m=n$. In particular, the estimate (3-8) improves (1-10) on complete Riemannian manifolds.

Theorem 3.6. Let $\left(M^{n}, g\right)$ and $K$ be as in Theorem 3.1. Let $u$ be a positive solution to the porous medium equation (1-8) with $p>1$. Let $v=(p /(p-1)) u^{p-1}$ and $M=(p-1) \max _{B_{p}(2 R) \times[0, T]} v$. Then, on $B_{p}(R)$, we have

$$
\frac{|\nabla v|^{2}}{v}-\alpha(t) \frac{v_{t}}{v}-\varphi(t) \leq \tilde{a} M \frac{C(m)}{R^{2}}\left(1+\sqrt{K} R \operatorname{coth}(\sqrt{K} R)+\frac{\tilde{a} p^{2}}{(p-1) \tanh (M K t)}\right),
$$

where $\alpha(t), \varphi(t)$ are given by

$$
\begin{aligned}
\varphi(t) & =\tilde{a} M K(\operatorname{coth}(M K t)+1), \\
\alpha(t) & =1+\frac{\cosh (M K t) \sinh (M K t)-M K t}{\sinh ^{2}(M K t)} .
\end{aligned}
$$

Taking $R \rightarrow \infty$, we thus obtain the following estimate on $\left(M^{n}, g\right)$ :

$$
\frac{|\nabla v|^{2}}{v}-\alpha(t) \frac{v_{t}}{v}-\varphi(t) \leq 0
$$

Corollary 3.7. Let $\left(M^{n}, g\right)$ be a complete noncompact Riemannian manifold with $\operatorname{Ric}_{\phi}^{m} \geq-K$, where $K$ is a nonnegative constant. Let $u$ be a positive solution to $(1-8)$ with $p>1$. Let $v=(p /(p-1)) u^{p-1}$ and $M=(p-1) \sup _{M^{n} \times[0, T]} v$, $\widetilde{M}=\inf _{M^{n} \times[0, T]} v$. Then, for any $x_{1}, x_{2} \in M^{n}, 0<t_{1}<t_{2}<T, \alpha>1$, we have

$$
v\left(x_{1}, t_{1}\right) \leq v\left(x_{2}, t_{2}\right) A_{1}\left(t_{1}, t_{2}\right) \exp \left(\frac{\operatorname{dist}^{2}\left(x_{2}, x_{1}\right)}{4 \widetilde{M}\left(t_{2}-t_{1}\right)}\left(1+A_{2}\left(t_{1}, t_{2}\right)\right)\right),
$$

where $\operatorname{dist}\left(x_{2}, x_{1}\right)$ is the distance between $x_{1}$ and $x_{2}$ and

$$
\begin{aligned}
& A_{1}\left(t_{1}, t_{2}\right)=\left(\frac{\exp \left(2 M K t_{2}\right)-2 M K t_{2}-1}{\exp \left(2 M K t_{1}\right)-2 M K t_{1}-1}\right)^{\tilde{a} / 2}, \\
& A_{2}\left(t_{1}, t_{2}\right)=\frac{t_{2} \operatorname{coth}\left(M K t_{2}\right)-t_{1} \operatorname{coth}\left(M K t_{1}\right)}{t_{2}-t_{1}} .
\end{aligned}
$$

Theorem 3.8. Let $\left(M^{n}, g\right)$ and $K$ be as in Theorem 3.1. Let $u$ be a positive solution to the porous medium equation (1-8) with $p>1$. Let $v=(p /(p-1)) u^{p-1}$ and $M=(p-1) \max _{B_{p}(2 R) \times[0, T]} v$. Then, on $B_{p}(R)$, we have

$$
\begin{aligned}
\frac{|\nabla v|^{2}}{v}-\alpha(t) \frac{v_{t}}{v} & -\varphi(t) \\
& \leq \tilde{a} \alpha^{2}(t) M \frac{C(m)}{R^{2}}\left(1+\sqrt{K} R \operatorname{coth}(\sqrt{K} R)+\frac{\tilde{a} p^{2} \alpha^{2}(t)}{(p-1) \tanh (M K t)}\right),
\end{aligned}
$$


where

$$
\varphi(t)=\frac{\tilde{a}}{t}+\tilde{a} M K+\frac{\tilde{a}}{3}(M K)^{2} t \quad \text { and } \quad \alpha(t)=1+\frac{2}{3} M K t .
$$

Taking $R \rightarrow \infty$, we thus obtain the following estimate on $\left(M^{n}, g\right)$ :

$$
\frac{|\nabla v|^{2}}{v}-\alpha(t) \frac{v_{t}}{v}-\varphi(t) \leq 0
$$

Corollary 3.9. Let $\left(M^{n}, g\right)$ be a complete noncompact Riemannian manifold with $\operatorname{Ric}_{\phi}^{m} \geq-K$, where $K$ is a nonnegative constant. Let $u$ be a positive solution to (1-8) with $p>1$. Set

$$
v=p /(p-1) u^{p-1}, \quad M=(p-1) \sup _{M^{n} \times[0, T]} v, \quad \widetilde{M}=\inf _{M^{n} \times[0, T]} v .
$$

Then, for any $x_{1}, x_{2} \in M^{n}, 0<t_{1}<t_{2}<T, \alpha>1$, we have

$$
\begin{aligned}
v\left(x_{1}, t_{1}\right) \leq v\left(x_{2}, t_{2}\right) & \left(\frac{t_{2}}{t_{1}}\right)^{\tilde{a}}\left(\frac{1+\frac{2}{3} M K t_{2}}{1+\frac{2}{3} M K t_{1}}\right)^{-\tilde{a} / 4} \\
& \times \exp \left(\frac{\operatorname{dist}^{2}\left(x_{2}, x_{1}\right)}{4 \widetilde{M}\left(t_{2}-t_{1}\right)}\left(1+\frac{1}{3} M K\left(t_{2}+t_{1}\right)\right)+\frac{\tilde{a}}{2} M K\left(t_{2}-t_{1}\right)\right),
\end{aligned}
$$

where $\operatorname{dist}\left(x_{2}, x_{1}\right)$ is the distance between $x_{1}$ and $x_{2}$.

Remark 3.10. Our Theorems 3.6 and 3.8 reduce to Theorems 1.3 and 1.4 from [Huang et al. 2013], respectively, by taking $m=n$. Moreover, when $t$ is small enough, $\alpha(t)$ and $\varphi(t)$ defined by (3-10) and (3-12) both satisfy $\alpha(t) \rightarrow 1$ and $\varphi(t) \leq 2 \tilde{a} M K+\tilde{a} / t$. Hence (3-11) and (3-13) show

$$
\frac{|\nabla v|^{2}}{v}-\alpha(t) \frac{v_{t}}{v} \leq 2 \tilde{a} M K+\frac{\tilde{a}}{t} .
$$

Clearly, for $t$ small enough, (3-14) is better than (1-10). In this sense, (3-11) and (3-13) improve (1-10) on complete Riemannian manifolds.

\section{Proofs of Theorems 1.9 and 1.10}

Lemma 4.1. If $M^{n}$ is a compact Riemannian manifold and $u$ is a positive solution to $(1-8)$ with $p \neq 0$, then

$$
\frac{d}{d t} \int_{M^{n}} u v d \mu=(p-1) \int_{M^{n}}\left(\Delta_{\phi} v\right) u v d \mu=-p \int_{M^{n}}|\nabla v|^{2} u d \mu .
$$

Proof. From (2-1), we have $(u v)_{t}=v u_{t}+u v_{t}=v \Delta_{\phi}\left(u^{p}\right)+(p-1) u v \Delta_{\phi} v+u|\nabla v|^{2}$. It follows from $\nabla\left(u^{p}\right)=u \nabla v$ that

$$
\int_{M^{n}}\left[v \Delta_{\phi}\left(u^{p}\right)+u|\nabla v|^{2}\right] d \mu=\int_{M^{n}}\left[-\nabla v \nabla\left(u^{p}\right)+u|\nabla v|^{2}\right] d \mu=0 .
$$


Hence

$$
\begin{aligned}
\frac{d}{d t} \int_{M^{n}} u v d \mu & =\int_{M^{n}}(u v)_{t} d \mu=\int_{M^{n}}\left[v \Delta_{\phi}\left(u^{p}\right)+(p-1) u v \Delta_{\phi} v+u|\nabla v|^{2}\right] d \mu \\
& =(p-1) \int_{M^{n}}\left(\Delta_{\phi} v\right) u v d \mu=p \int_{M^{n}}\left(\Delta_{\phi} v\right) u^{p} d \mu \\
& =-p \int_{M^{n}} \nabla v \nabla\left(u^{p}\right) d \mu=-p \int_{M^{n}}|\nabla v|^{2} u d \mu .
\end{aligned}
$$

Lemma 4.2. If $M^{n}$ is a compact Riemannian manifold and $u$ is a positive solution to $(1-8)$ with $p \neq 0$, then

$$
\frac{d}{d t} \int_{M^{n}}\left(\Delta_{\phi} v\right) u v d \mu=2 \int_{M^{n}}\left[(p-1)\left(\Delta_{\phi} v\right)^{2}+\left|\nabla^{2} v\right|^{2}+\operatorname{Ric}_{\phi}(\nabla v, \nabla v)\right] u v d \mu
$$

Proof. Noticing that

$$
\frac{d}{d t} \int_{M^{n}}\left(\Delta_{\phi} v\right) u v d \mu=\int_{M^{n}}\left[\left(\Delta_{\phi} v\right)_{t} u v+\left(\Delta_{\phi} v\right)(u v)_{t}\right] d \mu
$$

a direct calculation gives

$\left(\Delta_{\phi} v\right)_{t}$

$$
\begin{aligned}
& =\Delta_{\phi}\left[(p-1) v \Delta_{\phi} v+|\nabla v|^{2}\right] \\
& =(p-1)\left[\left(\Delta_{\phi} v\right)^{2}+2 \nabla v \nabla \Delta_{\phi} v+v \Delta_{\phi}^{2} v\right]+\Delta_{\phi}|\nabla v|^{2} \\
& =(p-1)\left(\Delta_{\phi} v\right)^{2}+2 p \nabla v \nabla \Delta_{\phi} v+(p-1) v \Delta_{\phi}^{2} v+2\left[\left|\nabla^{2} v\right|^{2}+\operatorname{Ric}_{\phi}(\nabla v, \nabla v)\right] .
\end{aligned}
$$

We derive from $(p-1) \nabla\left(u v^{2}\right)=(2 p-1) u v \nabla v$ that

$$
\begin{aligned}
\int_{M^{n}}\left[2 p \nabla v \nabla \Delta_{\phi} v+(\right. & \left.p-1) v \Delta_{\phi}^{2} v\right] u v d \mu \\
& =\int_{M^{n}} 2 p \nabla v \nabla\left(\Delta_{\phi} v\right) u v d \mu-\int_{M^{n}}(p-1) \nabla\left(u v^{2}\right) \nabla \Delta_{\phi} v d \mu \\
& =\int_{M^{n}} \nabla v \nabla\left(\Delta_{\phi} v\right) u v d \mu .
\end{aligned}
$$

Hence

$$
\begin{aligned}
& \int_{M^{n}}\left(\Delta_{\phi} v\right)_{t} u v d \mu \\
& \quad=\int_{M^{n}}\left\{(p-1)\left(\Delta_{\phi} v\right)^{2}+\nabla v \nabla \Delta_{\phi} v+2\left[\left|\nabla^{2} v\right|^{2}+\operatorname{Ric}_{\phi}(\nabla v, \nabla v)\right]\right\} u v d \mu .
\end{aligned}
$$


On the other hand,

$$
\begin{aligned}
\int_{M^{n}} \Delta_{\phi} v & (u v)_{t} d \mu \\
& =\int_{M^{n}} \Delta_{\phi} v\left[v \Delta_{\phi}\left(u^{p}\right)+(p-1) u v \Delta_{\phi} v+u|\nabla v|^{2}\right] d \mu \\
& =\int_{M^{n}}\left[-\nabla\left(v \Delta_{\phi} v\right) \nabla\left(u^{p}\right)+(p-1) u v\left(\Delta_{\phi} v\right)^{2}+u|\nabla v|^{2} \Delta_{\phi} v\right] d \mu \\
& =\int_{M^{n}}\left[-\nabla\left(v \Delta_{\phi} v\right) u \nabla v+(p-1) u v\left(\Delta_{\phi} v\right)^{2}+u|\nabla v|^{2} \Delta_{\phi} v\right] d \mu \\
& =\int_{M^{n}}\left[-\nabla v \nabla \Delta_{\phi} v+(p-1)\left(\Delta_{\phi} v\right)^{2}\right] u v d \mu .
\end{aligned}
$$

Inserting (4-3) and (4-4) into (4-2) concludes the proof of Lemma 4.2

Proof of Theorems 1.9 and 1.10. By Lemma 4.1, we have

$$
\begin{aligned}
\frac{d}{d t} \mathcal{N}_{p, m}(g, u, t) & =-\tilde{a} t^{\tilde{a}-1} \int_{M^{n}} u v d \mu-(p-1) t^{\tilde{a}} \int_{M^{n}}\left(\Delta_{\phi} v\right) u v d \mu \\
& =-t^{\tilde{a}} \int_{M^{n}}\left((p-1) \Delta_{\phi} v+\frac{\tilde{a}}{t}\right) u v d \mu .
\end{aligned}
$$

We obtain (1-29) and (1-32). On the other hand, from the definition of $\mathcal{W}_{p, m}(g, u, t)$ in (1-28), we have

$$
\begin{aligned}
\mathscr{W}_{p, m}(g, u, t) & =\frac{d}{d t}\left[t \mathcal{N}_{p, m}(g, u, t)\right] \\
& =\mathcal{N}_{p, m}(g, u, t)+t \frac{d}{d t} \mathcal{N}_{p, m}(g, u, t) \\
& =t^{\tilde{a}+1} \int_{M^{n}}\left(p \frac{|\nabla v|^{2}}{v}-\frac{\tilde{a}+1}{t}\right) u v d \mu,
\end{aligned}
$$

where Lemma 4.1 was used in the last equality. Hence we derive (1-30) and (1-33).

Notice that the estimate (1-10) also holds for compact Riemannian manifolds. Taking $K=0$ and then letting $\alpha \rightarrow 1$ in (1-10) yields

$$
(p-1) \Delta_{\phi} v+\frac{\tilde{a}}{t}=\frac{v_{t}}{v}-\frac{|\nabla v|^{2}}{v}+\frac{\tilde{a}}{t} \geq 0,
$$

which allows us to concludes that if $\operatorname{Ric}_{\phi}^{m} \geq 0$, then $\mathcal{N}_{p, m}(g, u, t)$ is nonincreasing in $t$. When $p \in(1-2 / m, 1)$ and $\operatorname{Ric}_{\phi}^{m} \geq 0$, we also get from (1-12) that

$$
(p-1) \Delta_{\phi} v+\frac{\tilde{a}}{t}=\frac{v_{t}}{v}-\frac{|\nabla v|^{2}}{v}+\frac{\tilde{a}}{t} \leq 0,
$$

which shows that $\mathcal{N}_{p, m}(g, u, t)$ is also nonincreasing in $t$.

Now we are in a position to prove (1-31). From (1-29), we have 


$$
\begin{aligned}
& \frac{d}{d t}\left(t \frac{d}{d t} \mathcal{N}_{p, m}(g, u, t)\right) \\
& =\frac{d}{d t}\left(-t^{\tilde{a}+1} \int_{M^{n}}(p-1)\left(\Delta_{\phi} v\right) u v d \mu-\tilde{a} t^{\tilde{a}} \int_{M^{n}} u v d \mu\right) \\
& =\frac{d}{d t}\left(-t^{\tilde{a}+1} \int_{M^{n}}(p-1)\left(\Delta_{\phi} v\right) u v d \mu+\tilde{a} \mathcal{N}_{p, m}(g, u, t)\right) \\
& =-2 t^{\tilde{a}+1} \int_{M^{n}}\left((p-1)^{2}\left(\Delta_{\phi} v\right)^{2}+(p-1)\left|\nabla^{2} v\right|^{2}+(p-1) \operatorname{Ric}_{\phi}(\nabla v, \nabla v)\right) u v d \mu \\
& \quad-(\tilde{a}+1) t^{\tilde{a}} \int_{M^{n}}(p-1)\left(\Delta_{\phi} v\right) u v d \mu-\tilde{a} t^{\tilde{a}} \int_{M^{n}}\left((p-1) \Delta_{\phi} v+\frac{\tilde{a}}{t}\right) u v d \mu,
\end{aligned}
$$

where the last equality used Lemma 4.2. Hence

$$
\text { (4-5) } \begin{aligned}
& \frac{d}{d t} \mathscr{W}_{p, m}(g, u, t) \\
= & \frac{d}{d t}\left(t \frac{d}{d t} \mathcal{N}_{p, m}(g, u, t)+\mathcal{N}_{p, m}(g, u, t)\right) \\
= & -2 t^{\tilde{a}+1} \int_{M^{n}}\left[(p-1)^{2}\left(\Delta_{\phi} v\right)^{2}+(p-1)\left|\nabla^{2} v\right|^{2}+(p-1) \operatorname{Ric}_{\phi}(\nabla v, \nabla v)\right] u v d \mu \\
& -(\tilde{a}+1) t^{\tilde{a}} \int_{M^{n}}(p-1)\left(\Delta_{\phi} v\right) u v d \mu-(\tilde{a}+1) t^{\tilde{a}} \int_{M^{n}}\left((p-1) \Delta_{\phi} v+\frac{\tilde{a}}{t}\right) u v d \mu \\
= & -2 t^{\tilde{a}+1} \int_{M^{n}}\left((p-1)^{2}\left(\Delta_{\phi} v\right)^{2}+(p-1)\left|\nabla^{2} v\right|^{2}+(p-1) \operatorname{Ric}_{\phi}(\nabla v, \nabla v)\right. \\
& \left.+(p-1) \frac{\tilde{a}+1}{t} \Delta_{\phi} v+\frac{\tilde{a}^{2}+\tilde{a}}{2 t^{2}}\right) u v d \mu .
\end{aligned}
$$

Notice that

$$
\begin{aligned}
& (p-1)^{2}\left(\Delta_{\phi} v\right)^{2}+(p-1) \frac{\tilde{a}+1}{t} \Delta_{\phi} v+\frac{\tilde{a}^{2}+\tilde{a}}{2 t^{2}} \\
& =\left|(p-1) \Delta_{\phi} v+\frac{m(p-1)}{[m(p-1)+2] t}\right|^{2}+\frac{2(p-1)}{[m(p-1)+2] t} \Delta_{\phi} v+\frac{(p-1) m}{[m(p-1)+2]^{2} t^{2}},
\end{aligned}
$$

and hence

$$
\begin{array}{r}
(p-1)^{2}\left(\Delta_{\phi} v\right)^{2}+(p-1) \frac{\tilde{a}+1}{t} \Delta_{\phi} v+\frac{\tilde{a}^{2}+\tilde{a}}{2 t^{2}}+(p-1)\left|\nabla^{2} v\right|^{2}+\frac{p-1}{m-n}(\nabla \phi \nabla v)^{2} \\
=\left|(p-1) \Delta_{\phi} v+\frac{m(p-1)}{[m(p-1)+2] t}\right|^{2}+(p-1)\left|\nabla^{2} v+\frac{g}{[m(p-1)+2] t}\right|^{2} \\
+\frac{p-1}{m-n}\left|\nabla \phi \nabla v-\frac{m-n}{[m(p-1)+2] t}\right|^{2} .
\end{array}
$$


We complete the proof of (1-31) by putting (4-6) into (4-5).

When $p \in(0,1)$, by the Cauchy-Schwarz inequality, we have

$$
\begin{aligned}
&-(p-1)\left|\nabla^{2} v+\frac{g}{[m(p-1)+2] t}\right|^{2} \\
& \geq-\frac{p-1}{n}\left|\Delta v+\frac{n}{[m(p-1)+2] t}\right|^{2} \\
&=-\frac{1}{n(p-1)}\left|(p-1) \Delta_{\phi} v+\frac{\tilde{a}}{t}\right|^{2}-\frac{p-1}{n}\left|\nabla \phi \nabla v-\frac{m-n}{[m(p-1)+2] t}\right|^{2} \\
&-\frac{2}{n}\left((p-1) \Delta_{\phi} v+\frac{\tilde{a}}{t}\right)\left(\nabla \phi \nabla v-\frac{m-n}{[m(p-1)+2] t}\right) .
\end{aligned}
$$

Hence

$$
\begin{gathered}
-(p-1)\left|\nabla^{2} v+\frac{g}{[m(p-1)+2] t}\right|^{2}-\frac{p-1}{m-n}\left|\nabla \phi \nabla v-\frac{m-n}{[m(p-1)+2] t}\right|^{2} \\
\geq \frac{1-n(1-p)}{n(1-p)}\left|(p-1) \Delta_{\phi} v+\frac{\tilde{a}}{t}\right|^{2}+\frac{m(1-p)}{n(m-n)}\left|\nabla \phi \nabla v-\frac{m-n}{[m(p-1)+2] t}\right|_{\phi} v+\left.\frac{\tilde{a}}{t}\right|^{2} \\
-\frac{2}{n}\left((p-1) \Delta_{\phi} v+\frac{\tilde{a}}{t}\right)\left(\nabla \phi \nabla v-\frac{m-n}{[m(p-1)+2]}\right) \\
\geq\left(\frac{1-n(1-p)}{n(1-p)}-\frac{\varepsilon}{n}\right)\left|(p-1) \Delta_{\phi} v+\frac{\tilde{a}}{t}\right|^{2} \\
+\left(\frac{m(1-p)}{n(m-n)}-\frac{1}{n \varepsilon}\right)\left|\nabla \phi \nabla v-\frac{m-n}{[m(p-1)+2] t}\right|^{2},
\end{gathered}
$$

where $\varepsilon \geq m-n$ is a positive constant and satisfies

$$
1-1 /(n+\varepsilon) \leq p \leq 1-(m-n) /(m \varepsilon) .
$$

Inserting (4-7) into (1-31) gives

$$
\begin{aligned}
& \frac{d}{d t} W_{p, m}(g, u, t) \\
& \leq 2 t^{a+1} \int_{M^{n}}\left((1-p) \operatorname{Ric}_{\phi}^{m}(\nabla v, \nabla v)+\left(\frac{1-n(1-p)}{n(1-p)}-\frac{\varepsilon}{n}\right)\left|(p-1) \Delta_{\phi} v+\frac{\tilde{a}}{t}\right|^{2}\right. \\
& \left.+\left(\frac{m(1-p)}{n(m-n)}-\frac{1}{n \varepsilon}\right)\left|\nabla \phi \nabla v-\frac{m-n}{[m(p-1)+2] t}\right|^{2}\right) u v d \mu .
\end{aligned}
$$

This completes the proof of (1-34). 


\section{Acknowledgements}

The authors thank Professor Xiang-Dong Li for his valuable comments and Mr. Zhijie Huang for helpful discussions. The authors also thank the referee for careful reading and many valuable suggestions to improve readability.

\section{References}

[Aronson and Bénilan 1979] D. G. Aronson and P. Bénilan, "Régularité des solutions de l'équation des milieux poreux dans $\mathbb{R}^{N}$, C. R. Acad. Sci. Paris (A) 288:2 (1979), 103-105. MR 82i:35090 Zbl 0397.35034

[Bakry 1994] D. Bakry, "L'hypercontractivité et son utilisation en théorie des semigroupes", pp. 1-114 in Lectures on probability theory (Saint-Flour, 1992), edited by D. Bakry et al., Lecture Notes in Math. 1581, Springer, Berlin, 1994. MR 95m:47075 Zbl 0856.47026

[Bakry and Émery 1985] D. Bakry and M. Émery, "Diffusions hypercontractives", pp. 177-206 in Séminaire de probabilités, XIX, 1983/84, edited by J. Azéma and M. Yor, Lecture Notes in Math. 1123, Springer, Berlin, 1985. MR 88j:60131 Zbl 0561.60080

[Bakry and Qian 1999] D. Bakry and Z. M. Qian, "Harnack inequalities on a manifold with positive or negative Ricci curvature", Rev. Mat. Iberoamericana 15:1 (1999), 143-179. MR 2000f:58052 Zbl 0924.58096

[Bakry and Qian 2005] D. Bakry and Z. M. Qian, "Volume comparison theorems without Jacobi fields", pp. 115-122 in Current trends in potential theory (Bucharest, 2002/2003), edited by D. Bakry et al., Theta Ser. Adv. Math. 4, Theta, Bucharest, 2005. MR 2007e:58048 Zbl 1212.58019

[Calabi 1958] E. Calabi, "An extension of E. Hopf's maximum principle with an application to Riemannian geometry”, Duke Math. J. 25 (1958), 45-56. MR 19,1056e Zbl 0079.11801

[Cheng and Yau 1975] S. Y. Cheng and S.-T. Yau, "Differential equations on Riemannian manifolds and their geometric applications", Comm. Pure Appl. Math. 28:3 (1975), 333-354. MR 52 \#6608 Zbl 0312.53031

[Daskalopoulos and Kenig 2007] P. Daskalopoulos and C. E. Kenig, Degenerate diffusions: initial value problems and local regularity theory, EMS Tracts in Mathematics 1, European Mathematical Society, Zürich, 2007. MR 2009b:35214 Zbl 1205.35002

[Davies 1989] E. B. Davies, Heat kernels and spectral theory, Cambridge Tracts in Mathematics 92 , Cambridge University Press, 1989. MR 90e:35123 Zbl 0699.35006

[Ecker 2007] K. Ecker, “A formula relating entropy monotonicity to Harnack inequalities”, Comm. Anal. Geom. 15:5 (2007), 1025-1061. MR 2009c:53088 Zbl 1167.53055

[Fang et al. 2009] F. Fang, X.-D. Li, and Z. Zhang, "Two generalizations of Cheeger-Gromoll splitting theorem via Bakry-Emery Ricci curvature", Ann. Inst. Fourier (Grenoble) 59:2 (2009), 563-573. MR 2010b:53057 Zbl 1166.53023

[Hamilton 1993] R. S. Hamilton, "A matrix Harnack estimate for the heat equation", Comm. Anal. Geom. 1:1 (1993), 113-126. MR 94g:58215 Zbl 0799.53048

[Huang et al. 2013] G. Huang, Z. Huang, and H. Li, "Gradient estimates for the porous medium equations on Riemannian manifolds", J. Geom. Anal. 23:4 (2013), 1851-1875. MR 3107682 arXiv 1106.2373

[Kotschwar and Ni 2009] B. Kotschwar and L. Ni, "Local gradient estimates of $p$-harmonic functions, 1/H-flow, and an entropy formula", Ann. Sci. Éc. Norm. Supér. (4) 42:1 (2009), 1-36. MR 2010g:53121 Zbl 1182.53060 
[Ledoux 2000] M. Ledoux, "The geometry of Markov diffusion generators", Ann. Fac. Sci. Toulouse Math. (6) 9:2 (2000), 305-366. MR 2002a:58045 Zbl 0980.60097

[Li 2005] X.-D. Li, "Liouville theorems for symmetric diffusion operators on complete Riemannian manifolds”, J. Math. Pures Appl. (9) 84:10 (2005), 1295-1361. MR 2006f:58046 Zbl 1082.58036

[Li 2011] X.-D. Li, "Perelman's $W$-entropy for the Fokker-Planck equation over complete Riemannian manifolds", Bull. Sci. Math. 135:6-7 (2011), 871-882. MR 2012m:53077 Zbl 1230.82039

[Li 2012] X.-D. Li, "Perelman's entropy formula for the Witten Laplacian on Riemannian manifolds via Bakry-Emery Ricci curvature”, Math. Ann. 353:2 (2012), 403-437. MR 2915542 Zbl 06043348

[Li 2013] X.-D. Li, "Hamilton's Harnack inequality and the $W$-entropy formula on complete Riemannian manifolds", preprint, 2013. arXiv 1303.1242

[Li and Li 2013] S. Li and X.-D. Li, "Perelman's entropy formula for the Witten Laplacian on manifolds with time dependent metrics and potentials", preprint, 2013. arXiv 1303.6019

[Li and Xu 2011] J. Li and X. Xu, "Differential Harnack inequalities on Riemannian manifolds, I: Linear heat equation”, Adv. Math. 226:5 (2011), 4456-4491. MR 2012h:53079 Zbl 1226.58009

[Li and Yau 1986] P. Li and S.-T. Yau, "On the parabolic kernel of the Schrödinger operator", Acta Math. 156:3-4 (1986), 153-201. MR 87f:58156 Zbl 0611.58045

[Lu et al. 2009] P. Lu, L. Ni, J. L. Vázquez, and C. Villani, "Local Aronson-Bénilan estimates and entropy formulae for porous medium and fast diffusion equations on manifolds", J. Math. Pures Appl. (9) 91:1 (2009), 1-19. MR 2010j:35563 Zbl 1156.58015

[Ni 2002] L. Ni, "The Poisson equation and Hermitian-Einstein metrics on holomorphic vector bundles over complete noncompact Kähler manifolds", Indiana Univ. Math. J. 51:3 (2002), 679-704. MR 2003m:32021 Zbl 1035.53032

[Ni 2004a] L. Ni, "The entropy formula for linear heat equation", J. Geom. Anal. 14:1 (2004), 87-100. MR 2004m:53118a Zbl 1044.58030

[Ni 2004b] L. Ni, "Addenda to 'The entropy formula for linear heat equation"”, J. Geom. Anal. 14:2 (2004), 369-374. MR 2004m:53118b Zbl 1062.58028

[Perelman 2002] G. Perelman, "The entropy formula for the Ricci flow and its geometric applications", preprint, 2002. Zbl 1130.53001 arXiv math/0211159

[Qian 1997] Z. M. Qian, "Estimates for weighted volumes and applications", Quart. J. Math. Oxford Ser. (2) 48:190 (1997), 235-242. MR 98e:53058 Zbl 0902.53032

[Qian 1998] Z. M. Qian, "A comparison theorem for an elliptic operator", Potential Anal. 8:2 (1998), 137-142. MR 99d:58161 Zbl 0930.58012

[Vázquez 2006] J. L. Vázquez, Smoothing and decay estimates for nonlinear diffusion equations: equations of porous medium type, Oxford Lecture Series in Mathematics and its Applications 33, Oxford University Press, 2006. MR 2007k:35008 Zbl 1113.35004

[Vázquez 2007] J. L. Vázquez, The porous medium equation: mathematical theory, Clarendon, Oxford, 2007. MR 2008e:35003 Zbl 1107.35003

[Wang 1997] F.-Y. Wang, "Logarithmic Sobolev inequalities on noncompact Riemannian manifolds", Probab. Theory Related Fields 109:3 (1997), 417-424. MR 98i:58253 Zbl 0887.35012

[Wang 2004] F.-Y. Wang, "Equivalence of dimension-free Harnack inequality and curvature condition”, Integral Equations Operator Theory 48:4 (2004), 547-552. MR 2004m:58061 Zbl 1074.47020

[Wang and Chen 2013] Y. Wang and W. Chen, "Gradient estimates for weighted diffusion equations on smooth metric measure spaces”, J. Math. (Wuhan) 33:2 (2013), 248-258. MR 3076231 
[Wang et al. 2013] Y. Wang, J. Yang, and W. Chen, "Gradient estimates and entropy formulae for weighted $p$-heat equations on smooth metric measure spaces”, Acta Math. Sci. Ser. B Engl. Ed. 33:4 (2013), 963-974. MR 3072133

[Wei and Wylie 2009] G. Wei and W. Wylie, "Comparison geometry for the Bakry-Emery Ricci tensor”, J. Differential Geom. 83:2 (2009), 377-405. MR 2011a:53064 Zbl 1189.53036

[Xu 2012] X. Xu, "Gradient estimates for $u_{t}=\Delta F(u)$ on manifolds and some Liouville-type theorems", J. Differential Equations 252:2 (2012), 1403-1420. MR 2853544 Zbl 1235.53045

[Yau 1994] S.-T. Yau, "On the Harnack inequalities of partial differential equations", Comm. Anal. Geom. 2:3 (1994), 431-450. MR 96f:58186 Zbl 0841.58059

[Yau 1995] S.-T. Yau, "Harnack inequality for non-self-adjoint evolution equations", Math. Res. Lett. 2:4 (1995), 387-399. MR 96k:58211 Zbl 0884.58091

Received December 10, 2012. Revised May 13, 2013.

GUANGYUE HUANG

College of Mathematics AND InFORMation SCIENCE

HENAN NORMAL UNIVERSITY

XINXIANG 453007

CHINA

hgy@henannu.edu.cn

HAIZHONG LI

DEPARTMENT OF MATHEMATICAL SCIENCES

TSINGHUA UNIVERSITY

BEIJING 100084

CHINA

hli@math.tsinghua.edu.cn 


\title{
PACIFIC JOURNAL OF MATHEMATICS
}

\author{
msp.org/pjm
}

Founded in 1951 by E. F. Beckenbach (1906-1982) and F. Wolf (1904-1989)

\section{EDITORS}

V. S. Varadarajan (Managing Editor)

Department of Mathematics

University of California

Los Angeles, CA 90095-1555

pacific@math.ucla.edu

Paul Balmer

Department of Mathematics

University of California

Los Angeles, CA 90095-1555

balmer@math.ucla.edu

Daryl Cooper

Department of Mathematics

University of California

Santa Barbara, CA 93106-3080 cooper@math.ucsb.edu

Jiang-Hua $\mathrm{Lu}$

Department of Mathematics

Pokfulam Rd., Hong Kong jhlu@maths.hku.hk
The University of Hong Kong

Don Blasius

Department of Mathematics University of California

Los Angeles, CA 90095-1555

blasius@math.ucla.edu

Robert Finn

Department of Mathematics Stanford University

Stanford, CA 94305-2125

finn@math.stanford.edu

Sorin Popa

Department of Mathematics

University of California

Los Angeles, CA 90095-1555 popa@math.ucla.edu

Paul Yang

Department of Mathematics Princeton University

Princeton NJ 08544-1000

yang@math.princeton.edu

\section{PRODUCTION}

Silvio Levy, Scientific Editor, production@msp.org

\section{SUPPORTING INSTITUTIONS}

ACADEMIA SINICA, TAIPEI

CALIFORNIA INST. OF TECHNOLOGY

INST. DE MATEMÁTICA PURA E APLICADA

KEIO UNIVERSITY

MATH. SCIENCES RESEARCH INSTITUTE

NEW MEXICO STATE UNIV.

OREGON STATE UNIV.

\author{
STANFORD UNIVERSITY \\ UNIV. OF BRITISH COLUMBIA \\ UNIV. OF CALIFORNIA, BERKELEY \\ UNIV. OF CALIFORNIA, DAVIS \\ UNIV. OF CALIFORNIA, LOS ANGELES \\ UNIV. OF CALIFORNIA, RIVERSIDE \\ UNIV. OF CALIFORNIA, SAN DIEGO \\ UNIV. OF CALIF., SANTA BARBARA
}

\author{
Vyjayanthi Chari \\ Department of Mathematics \\ University of California \\ Riverside, CA 92521-0135 \\ chari@math.ucr.edu \\ Kefeng Liu \\ Department of Mathematics \\ University of California \\ Los Angeles, CA 90095-1555 \\ liu@math.ucla.edu \\ Jie Qing \\ Department of Mathematics \\ University of California \\ Santa Cruz, CA 95064 \\ qing@cats.ucsc.edu
}

These supporting institutions contribute to the cost of publication of this Journal, but they are not owners or publishers and have no responsibility for its contents or policies.

See inside back cover or msp.org/pjm for submission instructions.

The subscription price for 2014 is US \$410/year for the electronic version, and \$535/year for print and electronic.

Subscriptions, requests for back issues and changes of subscribers address should be sent to Pacific Journal of Mathematics, P.O. Box 4163, Berkeley, CA 94704-0163, U.S.A. The Pacific Journal of Mathematics is indexed by Mathematical Reviews, Zentralblatt MATH, PASCAL CNRS Index, Referativnyi Zhurnal, Current Mathematical Publications and Web of Knowledge (Science Citation Index).

The Pacific Journal of Mathematics (ISSN 0030-8730) at the University of California, c/o Department of Mathematics, 798 Evans Hall \#3840, Berkeley, CA 94720-3840, is published twelve times a year. Periodical rate postage paid at Berkeley, CA 94704, and additional mailing offices. POSTMASTER: send address changes to Pacific Journal of Mathematics, P.O. Box 4163, Berkeley, CA 94704-0163.

PJM peer review and production are managed by EditFLOW ${ }^{\circledR}$ from Mathematical Sciences Publishers.

\section{PUBLISHED BY}

mathematical sciences publishers

nonprofit scientific publishing

http://msp.org/

(C) 2014 Mathematical Sciences Publishers 


\section{PACIFIC JOURNAL OF MATHEMATICS}

Volume $268 \quad$ No. $1 \quad$ March 2014

AlEXANDRE PAIVA BARRETO

A transport inequality on the sphere obtained by mass transport

DARIO CORDERO-ERAUSQUIN

A cohomological injectivity result for the residual automorphic spectrum of $\mathrm{GL}_{n}$

HARALD GROBNER

Gradient estimates and entropy formulae of porous medium and fast diffusion equations for the Witten Laplacian

GUANGYUE HUANG and HAIZHONG LI

Controlled connectivity for semidirect products acting on locally finite trees

KeITH JoNES

An indispensable classification of monomial curves in $\mathbb{A}^{4}(\mathbb{k})$

ANARGYROS KATSABEKIS and IGNACIO OJEDA

Contracting an axially symmetric torus by its harmonic mean curvature

CHRISTOPHER KIM

Composition operators on strictly pseudoconvex domains with smooth symbol

HYUNGWOON KOO and SONG-YING LI

The Alexandrov problem in a quotient space of $\mathbb{U}^{2} \times \mathbb{R}$

AnA Menezes

Twisted quantum Drinfeld Hecke algebras

DEEPAK NAIDU

$L^{p}$ harmonic 1-forms and first eigenvalue of a stable minimal hypersurface

KEOMKYO SEO

Reconstruction from Koszul homology and applications to module and derived categories

RYO TAKAHASHI

A virtual Kawasaki-Riemann-Roch formula 